\title{
Manifold Methods for Assimilating Geophysical and Meteorological Data in Earth System Models and their Components
}

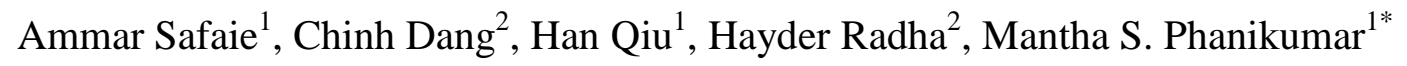

\begin{abstract}
A novel manifold method of reconstructing dynamically evolving spatial fields is presented for assimilating data from sensor networks in integrated land surface - subsurface, oceanic / lake models. The method was developed based on the assumption that data can be mapped onto an underlying differential manifold. In this study, the proposed method was used to reconstruct meteorological forcing over Lake Michigan, the bathymetry of an inland lake (Gull Lake), and precipitation over the Grand River watershed in Michigan. In the first case study, hourly interpolated meteorological forcing data were used to run a three-dimensional hydrodynamic model of Lake Michigan to quantify the improvement that results from the use of the new interpolation method. In the second example, the bathymetry of Gull Lake was reconstructed from measured scatter point data using the manifold technique. A hydrodynamic model of Gull Lake was developed and further improved using improved bathymetry. In the last case study, daily participation data over a six-year period were interpolated over the Grand River watershed and used as input to an integrated, distributed hydrologic model. All three examples illustrate the superior performance of the manifold method over standard methods in terms of accuracy and computational efficiency. Our results also indicate that evaluating the relative performance of interpolation methods using the cross-validation method can lead to misleading conclusions about the relative performance of the methods.
\end{abstract}




\section{$31 \quad 1 \quad$ Introduction}

32 Water continuously circulates between the Earth's surface and the atmosphere and moves

33 through watersheds to become stored underground as groundwater or in surface reservoirs such

34 as lakes. This circulation is a key aspect of Earth system models or components thereof and

35 meteorological forcing plays an important role in modeling coupled physical - biogeochemical

36 processes. Process-based modeling approaches describe land-lake-atmosphere interactions by

37 explicitly considering the spatiotemporal variability of meteorological forcing fields (Xue et al.,

38 2015). There are a variety of numerical ocean models, such as POM (Blumberg, and Mellor,

39 1987), ROMS (Haidvogel and Beckmann, 1999), and FVCOM (Chen et al., 2006), and their

40 performance is highly dependent on how realistic the distributions of surface forcing fields (wind

41 stress, heat flux, precipitation, evaporation) are. These forcing fields can be obtained from

42 observational data, the output of a weather forecast model, or a combination thereof (Xue et al.,

43 2015). In all cases it is crucial to use a suitable interpolation method in order to assimilate

44 observations into the models, and estimate variables at unsampled locations and/or times. For

45 instance, currents in large lakes such as the Laurentian Great Lakes are mostly controlled by

46 wind. Therefore, by improving the representation of wind fields in models of lake circulation, we

47 expect to describe coupled biophysical processes in lakes more accurately. For example, Safaie

48 et al. (2016) demonstrated that improved representation of meteorological fields based on natural

49 neighbor interpolation of weather station data produced superior results for currents and bacterial

50 concentrations relative to similar results based on a nearest neighbor interpolation of the same

51 data. 
52 In situ observations generally have sparse and inhomogeneous distribution in space and time,

53 and it is often infeasible to accurately reconstruct the true field from the data. However, more

54 information about the structure of the field and its evolution, allows for better approximations

55 (Barth et al., 2008). Various deterministic [e.g., nearest neighbor, natural neighbor, inverse

56 distance weighting (IDW), spline, polynomial] and geostatistical (e.g. kriging) interpolation

57 methods have been developed to generate spatial fields. There have been numerous efforts to

58 compare different spatial interpolation methods in order to identify the best method for a given

59 model application. Many researches have used cross-validation for assessing the performance of

60 the interpolation methods. In this method, a subset of the original dataset is withheld to be used

61 later for validating the interpolated field constructed from the rest of the observational data.

62 Mean error (ME), root mean square error (RMSE) and the coefficient of determination $\left(\mathrm{R}^{2}\right)$ are

63 commonly used to evaluate the performance of each interpolation method (Suparta and Rahman,

64 2016). However, every problem has a unique method of interpolation that works best for a given

65 distribution of observations and the intended use of the interpolated data. Density of a sensor

66 network, spatial variability of the variable of interest and its distribution, and observational

67 errors, all influence the accuracy of the interpolated field (MacEachren and Davidson, 1987). For

68 example, Luo et al. (2008) compared seven spatial interpolation techniques to identify which

69 method produced the best estimation of the wind speed data recorded across England and Wales.

70 Their study showed that kriging is the best method, and that the thin plate spline method had

71 higher ME and RMSE values. However, in (Suparta and Rahman, 2016) the performance of the

72 thin plate spline interpolation based on the RMSE and $\mathrm{R}^{2}$ values was found to be better than

73 kriging for less dense data points over the selected interpolation surface. Therefore, comparing

74 interpolation methods using the cross-validation method without considering the data structure 
75 and the purpose of interpolation is not guaranteed to produce the best representation of the 76 underlying data.

77 Precipitation is another important component of the water cycle. Spatial distribution of 78 precipitation influences the hydrologic response of a watershed (Daly et al., 2002). Basin 79 responses of rainfall-runoff processes are closely related to the spatial variability of precipitation 80 (Anquetin et al., 2010; Bell and Moore, 2000; Beven and Hornberger, 1982; Obled et al., 1994; 81 Schuurmans and Bierkens, 2007; Syed et al., 2003; Tetzlaff and Uhlenbrook, 2005).

82 Hydrologists usually use rain gauge data to obtain rainfall patterns over a watershed. Bell and 83 Moore (2000) found that the responses of a distributed hydrologic model are sensitive to the 84 locations of the rain gauges within the catchment and hence to the spatial variability of rainfall.

85 Nicótina et al. (2008) performed numerical experiments to study the effects of different spatial 86 resolutions of rainfall on various catchments. They found that the catchment response is sensitive 87 to the spatial distribution of rainfall only when water residence time in the channels is 88 comparable to the hillslope travel time; thus rainfall spatial heterogeneity likely plays a more 89 important role in affecting the runoff response of large watersheds (typically larger than 103 $90 \mathrm{~km}^{2}$ ) than smaller watersheds.

91 Various efforts have been devoted to improving the representation of spatially-distributed 92 rainfall fields in hydrologic modeling. For example, Ly et al. (2011) compared seven 93 interpolation methods for daily rainfall and found that geostatistical methods such as kriging and

94 IDW algorithms significantly outperformed the Thiessen polygon method, which is also known 95 as the nearest neighbor method. Sun et al. (2000) tested different rainfall estimation methods on 96 a $1060 \mathrm{~km}^{2}$ catchment in Australia to evaluate the flood modeling capabilities of a hydrologic 97 model and found that blending radar and rain gauge data in a co-kriging framework provided 
98 better performance compared with an approach based on kriging of the rain gauges data alone.

99 Masih et al. (2011) used a semi-distributed model, Soil Water Assessment Tool (SWAT), to

100 simulate the Karkheh River basin in Iran with two different precipitation interpolation methods.

101 Their results, based on a comparison of simulated and observed discharges using the metrics of 102 coefficient of determination and the Nash-Sutcliffe efficiency (NASH), showed that precipitation 103 interpolation with inverse distance and elevation weighting technique produced better 104 performance relative to the SWAT default method of nearest neighbor method.

105 Accurate representation of geophysical features such as topography and bathymetry is also 106 important in earth system models and their components, and model performance depends on the 107 interpolation method used to assign the topographic information over a numerical mesh in 108 processed-based models. Yan et al. (2014) compared different interpolation methods, including 109 IDW, global polynomial interpolation, local polynomial interpolation, radial basis functions, 110 ordinary kriging (OK), simple kriging (SK), universal kriging (UK), and co-kriging (CK) to 111 determine the water/land boundary point elevation based on in situ water level data from 14 112 control stations in Dongting Lake. They used a cross-validation method to select the optimal 113 method, which was found to be the OK method. Merwade (2009) studied the effect of spatial 114 trend on interpolation of river bathymetry, and compared the performance of different 115 interpolation methods. The number of measurements and their spatial arrangement, as well as 116 channel morphology and geology were found to influence the accuracy of the interpolation 117 results (Merwade, 2009). Due to the effects of these and other factors on the performance of 118 various methods, comparisons of different spatial interpolation methods could not point out the 119 best universal interpolation method (Li and Heap, 2008; Šiljeg et al., 2015). 
120 In this paper, we propose a novel manifold method to assimilate different types of 121 spatiotemporal data in integrated earth system models based on the hypothesis that an 122 environmental dataset (including independent variables such as longitude, latitude, and time, and 123 the measured variables of interest) can be mapped onto an underlying differential manifold. A 124 manifold $(M)$ is an $n$-dimensional topological space such that each point of $M$ and its 125 neighborhood can be approximated by a small flat piece in the Euclidean space, $\mathbb{R}^{n}$. We can 126 think of a manifold as a set of low-dimensional curves and surfaces within higher dimension 127 Euclidean spaces (Victor and Pollack, 2010). Some typical examples of manifolds are smooth 128 surfaces, such as a torus (Fig. 1a) or a sphere (Fig. 1b), where each point and its neighborhood 129 can be approximated by a small flat linear-subspace within the three-dimensional Euclidean 130 space. Another example of a manifold in a high dimensional space is a Calabi-Yau manifold 131 which has found important applications in theoretical physics (e.g. superstring theory). Fig. 1c 132 shows a two-dimensional cross-section of a six-dimensional Calabi-Yau manifold. Surfaces of 133 all these three manifolds are not a Euclidean space. The laws of the Euclidean geometry, 134 however, are valid locally.

135 Working directly in the high dimensional space generally involves dealing with complex 136 algorithms. Modeling the high dimensional data using manifolds with fewer degrees of freedom 137 has captured a great deal of attention recently (Zhang et al., 2016). The use of low-dimensional 138 manifolds not only reduces computational load for further processing, but also helps visualize the 139 entire dataset, which is an important initial step to make sense of the data before proceeding with 140 more goal-directed modeling and analyses (Ma et al., 2011).

141 The classic method of principal component analysis (PCA), including the more recent Robust 142 PCA (Candès et al., 2011), is arguably the most popular framework for approximating a set of 
143 high-dimensional data points by a low dimensional linear space. PCA does not work well when 144 the underlying data structure is non-linear. Under such scenario, PCA methods require 145 approximating the input data points using higher dimensional linear spaces to reduce the 146 approximation error. Instead of assuming that the input data follow a linear structure, an 147 alternative solution is learning the true underlying low-dimensional structure of the data. The 148 problem of non-linear dimensionality reduction for a set of high dimensional data points is 149 known as manifold learning. Examples of early works for non-linear dimensionality reduction 150 include Isomap (Tenenbaum et al., 2000), local linear embedding (LLE) (Roweis, 2000), and 151 Eigenmaps (Belkin and Niyogi, 2003), which have been used to learn the manifold structure of 152 data. Since then, the manifold model has been exploited extensively in numerous applications 153 such as face recognition, action classification, segmentation, image denoising, image/video 154 super-resolution, and multi-scale image analysis (Carin et al., 2011; Dang et al., 2013, 2014; 155 Dang and Radha, 2015).

156 Most of the above manifold learning methods have been inspired by linear techniques, mainly 157 based on the assumption that non-linear manifolds can be approximated by locally linear parts 158 (Mordohai and Medioni, 2010). Two pioneering works in this area are the Isomap approach 159 (Tenenbaum et al., 2000) and the LLE algorithm (Roweis, 2000). The Isomap algorithm aims to 160 preserve the geodesic distance among points from the input dataset. On the other hand, the LLE 161 algorithm targets the local linear geometry of neighbors in a manifold. Numerous works on 162 manifold learning have been further developed. A comprehensive review of prior works can be 163 found in (van der Maaten et al., 2009).

164 Data assimilation methods seek to estimate both model parameters and model states and 165 significant progress has been made in the development of joint state-parameter estimation 
methods such as the ensemble Kalman filter (EnKF) and its variants (Moradkhani et al., 2005;

167 Evensen, 2006; Pathiraja et al., 2016). The parameters estimated using these approaches are 168 usually time-dependent although most dynamical models used in earth sciences use time169 invariant parameters. Yang et al. (2007) describe an alternative optimization-assimilation 170 approach for soil moisture in which they first estimate parameters within a long optimization 171 window and then estimate model states within a short assimilation window. Due to the 172 computational nature of the three-dimensional process-based models considered in the present 173 study, we do not focus on the use of methods similar to the EnKF method. Instead we estimate 174 optimal (relative to the observations) model parameters and states over the simulation period. 175 Future papers will focus on the use assimilation methods such as the EnKF with the framework 176 of manifold methods.

177 In this paper, the effectiveness of the presented manifold algorithm is evaluated through 178 assimilation of geophysical and meteorological data in integrated land surface - subsurface and 179 lake models, although the methods described are general and can be used in many other areas of 180 computational geosciences. We first apply the proposed method to reconstruct wind fields (time181 varying vector fields) over Lake Michigan. Since currents in Lake Michigan are primarily driven 182 by wind, we expect to improve the simulation of hydrodynamic and biophysical variables of 183 interest by improving the wind fields. Instead of relying on the cross-validation of interpolated 184 wind data, however, we use a well-tested hydrodynamic model of Lake Michigan and compare 185 current measurements with simulated currents to test the interpolation methods. The manifold 186 methods are used to reconstruct meteorological data including wind fields, cloud cover, dew 187 point, pressure, shortwave and longwave solar radiation, relative humidity, and air temperatures 188 for improved simulation of circulation in Lake Michigan. Then the method is applied to 
assimilate bathymetry data as a scalar field for use in a hydrodynamic model of Gull Lake - a

190 relatively large ( $8 \mathrm{~km}^{2}$ surface area) and deep (34 m maximum depth) clear water lake in the in

191 Kalamazoo County in southwestern Michigan. In the third example, time-dependent fields of

192 participation are interpolated over the Grand River watershed and used as input for an integrated,

193 land surface - subsurface processes model (PAWS+CLM; Shen and Phanikumar, 2010; Shen et

194 al., 2013). Grand River watershed is located in the middle of Michigan's Lower Peninsula and is

195 the second largest watershed in Michigan. In this example, the manifold method is tested using

196 stream discharge outputs of the PAWS+CLM model which has been tested in several catchments

197 in the past (Niu et al., 2014; Niu and Phanikumar, 2015; Shen et al., 2014, 2013).

1982 Materials and Methods

\section{$199 \quad 2.1 \quad$ Manifold approach}

200 Based on Einstein's theory of relativity, physical events are located on the continuum

201 (manifold) of space-time. Therefore, station locations and times of observations form a space-

202 time manifold viewed as a four-dimensional vector space. One way to handle spatiotemporal

203 interpolation problems, inspired by this concept, is to integrate space and time simultaneously

204 (Li and Revesz, 2004). An underlying assumption behind this approach is that time and space

205 dimensions can be treated as equally important ( $\mathrm{Li}$ et al., 2014). In order to add time as another

206 dimension of space, time values are needed to be scaled for a spatiotemporal dataset by a scaling

207 speed (Li et al., 2014; Schwab and Beletsky, 1998). For a point measurement, we can then define

208 a four-vector $P^{\mu}=(c t, \vec{x})$ where $c$ is a time scale, $t$ is the time coordinate and $\vec{x}$ is a three-

209 dimensional vector space. We assume that the set of high-dimensional data points $P$ (and the

210 estimated data points $P_{0}$ ) belongs to a differential manifold $M$, which may be curved and have 
211 a complicated topology, but the neighborhood of each point is approximately similar to a small

212 piece of Euclidean space (resembles $\mathbb{R}^{\mathrm{D}}$ ). Since a traditional distance measure is built upon the

213 geometry of Euclidean space, we adapt the calculation to a neighborhood or a small region of the

214 assumed manifold.

215 An example of a one-dimensional curve in Fig. 2 illustrates the general idea of the manifold 216 estimation approach. The set of points $P$ in Fig. 2 includes sample data points where we have 217 measured data as well as a point $P_{0}$ where data are missing. For example, in the context of the 218 wind field data, one full measurement (or data point) includes five components: time, longitude, 219 latitude, wind speed, and wind direction. The partially missing data point may contain known 220 components (time, longitude, latitude) and unknown or missing components (wind speed and 221 wind direction).

222 Consider a smooth $n$-dimensional manifold $M$ embedded in a $D$-dimensional Euclidean space.

223 Suppose that it is desired to estimate the wind field for a data point $P_{0} \in \mathbb{R}^{n}$ from a set of 224 training data points that belong to a manifold $M$. The space/time coordinates of the point (the 225 independent variables) are known, however, the data (the dependent variable) are missing. We 226 denote $P_{0}=\left[\begin{array}{c}P_{0}^{\mu} \\ P_{0}^{\nu}\end{array}\right] \in \mathbb{R}^{n}$ as the data point using the superscript $\mu$ to denote the independent 227 variables and the superscript $v$ to denote the dependent variable which is the missing component 228 of interest here. $P_{0}^{\mu} \in \mathbb{R}^{n_{\mu}}$ is the sub-vector of the known components, and $P_{0}^{\nu} \in \mathbb{R}^{n_{\nu}}$ $229\left(n_{\mu}+n_{\nu}=n\right)$ is the corresponding sub-vector (e.g., wind velocity vector) for the missing 230 component where $P_{0}^{\nu}=\vec{V}=(u, v)$ and $u, v$ are the orthogonal components of the wind velocity 
$231(\vec{V})$. The training data points, for example $P=\left\{P_{1}, P_{2}, \ldots, P_{7}\right\}$ in Fig. 2, also include the two 232 components $P_{i}=\left[\begin{array}{l}P_{i}^{\mu} \\ P_{i}^{\nu}\end{array}\right] \in \mathbb{R}^{n}$, but there is no missing component here since both dependent and 233 independent variables are assumed to be known at the nearby stations. Given a point $P_{0}^{\mu} \in \mathbb{R}^{n_{\mu}}$,

234 the algorithm locates a set of nearest points to $P_{0}^{\mu}$ based on the distances $d\left(P_{i}^{\mu}, P_{0}^{\mu}\right)$ between 235 pairs of points $P_{i}^{\mu}$ and $P_{0}^{\mu}$. In order to determine local neighbors of $P_{0}^{\mu}$, we can calculate the 236 distances between $P_{0}^{\mu}$ and either all other points within a fixed radius $\varepsilon$, or all of its $k$ nearest 237 neighbors (Tenenbaum et al., 2000). Then, a tangent space (linear subspace) of the manifold $M$ 238 at the point $P_{0}$ is created from the set of nearest points (Fig. 3a), denoted by $T_{P_{0}}(M)=\left[\begin{array}{c}T^{\mu} \\ T^{v}\end{array}\right]$ 239 where $T^{\mu}, T^{\nu}$ denote the tangent spaces for the independent and dependent variables in the data 240 at $P_{0}^{\mu}$ and $P_{0}^{v}$. Finally, the point $P_{0}=\left[\begin{array}{c}P_{0}^{\mu} \\ P_{0}^{v}\end{array}\right] \in \square^{n}$ will be located as the closest point that belongs 241 to that tangent space.

242 To represent the closest distance between a point and a tangent space, we use the Euclidean 243 distance of an orthogonal projection from that point to the tangent space. Since a tangent space is 244 a linear space (or affine space in a more general case), one point can orthogonally project into 245 that space. The question is how to define neighbors for each data point? The underlying idea is 246 how to define similarity distance among the training data points, and then the overall similarity 247 matrix. Several methods have been considered in the past, such as k-nearest neighbors (Press et 248 al., 2007), $\epsilon$-ball method (Allard et al., 2012) or the use of sparse representation theory (Dang et 249 al., 2014, 2013; Dang and Radha, 2015). To approximate the wind field, we do not focus on 
250 analyzing a predetermined set of tangent spaces as was done earlier (Dang et al., 2014, 2013),

251 but instead create a tangent space for each input data point as creating a tangent space for a given

252 input data leads to a better approximation of the manifold.

253 The estimation of $P_{0}^{\nu}$ is performed using the following steps:

254 1. Given a set of neighboring points, estimate the tangent space $T^{\mu}$ at the point of interest, $P_{0}$ :

255 Details of the method for creating a tangent space from a set of data points are described in

256 Appendix-A and in Dang et al. (2014). One simple method is to create a tangent space using

257 singular value decomposition (SVD, Press et al., 2007). By way of an example in Fig. 2, a

258 tangent space (the line $b$ ) is created for $P_{0}^{\mu}$ from a set of its neighboring points $\left(P_{5}^{\mu}\right.$ and $\left.P_{6}{ }^{\mu}\right)$.

259 This tangent space at $P_{0}^{\mu} \in M$ is denoted by $\mathrm{T}^{\mu}$.

260 2. Find the orthogonal projection of $P_{0}^{\mu}$ onto the tangent space:

261 The closest point $P^{\prime} \in \mathrm{T}^{\mu}$ to the given point $P_{0}^{\mu}$ is located at the intersection of the line $b$ and

262 the line perpendicular to it which passes through the point $P_{0}^{\mu} \cdot P^{\prime}$ which is a projection of $P_{0}^{\mu}$

263 onto the subspace $\mathrm{T}^{\mu}$ can be represented as an approximation of point $P_{0}^{\mu}$. The orthogonal

264 projection of vector point $P_{0}$ in a high-dimensional space onto a low-dimensional vector

265 subspace is given by:

266

$\prod_{\mathrm{T}^{\mu}}\left(P_{0}^{\mu}\right)=A\left(A^{T} A\right)^{-1} A^{T} P_{0}^{\mu}=A A^{+} P_{0}^{\mu}$

267 where $A=T^{\mu} \in \mathbb{R}^{D \times n}$ is a full rank matrix with $n<D$ containing the set of points on the

268 tangent space of $P_{0}^{\mu}$ and $\prod_{\mathrm{T}^{\mu}}\left(P_{0}^{\mu}\right)$ denotes the projection of $P_{0}^{\mu}$ onto the subspace $T^{\mu}$. This 
269 projection is derived from the solution of the normal equation $A^{T} A x=A^{T} P_{0}^{\mu}$ which is equivalent

270 to the associated least squares solution of $A x=P_{0}^{\mu}$. Due to the difficulty associated with inverting

271 a general matrix that may be singular or non-square depending on the number of neighboring

272 points selected in the manifold method, the problem (1) can be posed as a minimization problem

273 in which the Moore-Penrose pseudoinverse $A^{+}$(Golub and Van Loan, 2013) of the original

274 matrix $A$ is used. The pseudoinverse $A^{+}$generalizes the concept of matrix inverse and arises in

275 the minimum norm (that is, approximate as opposed to exact) or best-fit (in a least squares sense)

276 solution to a system of linear equations. The problem: $\underset{\mathbf{x}}{\operatorname{minimize}}\left\|A x-P_{0}^{\mu}\right\|_{2}$ has the solution

$277 x=A^{+} P_{0}^{\mu}$. The pseudoinverse can be computed using SVD as follows: if $A=U \Sigma V^{T}$, where

$278 U, V$ denote unitary matrices and $\Sigma$ is a diagonal matrix containing the singular values of $A$,

279 then $A^{+}=V \Sigma^{+} U^{T}$. We used the function pinv to compute the pseudoinverse in MATLAB.

280 3. Find a linear representation coefficient vector $\alpha$ of that projection onto the tangent space:

282 This coefficient is calculated by solving the following equation:

$283 \prod_{\mathrm{T}^{\mu}}\left(P_{0}^{\mu}\right)=A \cdot \alpha$

284 4. Estimate the missing components of the point $P_{0}\left(P_{0}^{\nu}\right)$ :

285 The last step is finding a point on the subspace $T^{\nu}$ that is closest (in norm) to the point $P_{0}$. In

286 order to do that, $T^{\nu}$ is projected using the projection coefficient $\alpha$ computed in step 3:

$287 \quad P_{0}^{\nu} \cong T^{\nu} \cdot \alpha$

288 The result of this projection is the closest point to $P_{0}^{\nu}$ that belongs to its subspace. In this 289 algorithm, high-dimensional coordinates of selected neighborhoods on the manifold are 
290 projected to a low-dimensional subspace. An alternative to this approach is to use kernel 291 regression to assign a weight to each neighbor based on the distance from $P_{0}^{\mu}$ (Fig. 3b). A 292 weight for each selected neighborhood can be computed using the following Gaussian kernel 293 function:

$294 \quad W_{i}=e^{-\frac{\left(P_{i}^{\mu}-P_{o}^{\mu}\right)^{2}}{2 \sigma^{2}}}, \sigma=\sqrt{\operatorname{var}\left(d\left(P_{i}^{\mu}, P_{o}^{\mu}\right)\right)}$

295 Examples of manifolds representing geophysical (bathymetry) and meteorological (wind) data

296 are shown in Figures 4(a) and (b). These figures support the assumption that the manifold can be 297 considered as being linear locally, but with complicated topology overall.

\subsection{Test case: Analytical function}

299 Before applying the manifold method to reconstruct complex geophysical and meteorological 300 data, we first evaluate the effectiveness of the method in reproducing an analytical function, 301 since errors can be computed relative to the known function values; therefore, the F7 function 302 suggested by Lazzaro and Montefusco (2002) and Renka and Brown (1999) is used:

$F 7(x, y)=2 \cos (10 x) \cdot \sin (10 y)+\sin (10 x \cdot y)$

306 where the domain of F7 is restricted to $0 \leq x \leq 1$ and $0 \leq y \leq 1$ (Fig. 5a). Three sets of sparse

307 random points from a normal distribution were generated in the domain with numbers of 308 sampling points of 30, 60, and 90. The F7 function was sampled randomly as shown in Fig. 5b 309 and the manifold method was tested by withholding one point at a time and estimating its 310 associated value from the remaining points using the manifold method, in addition to other 
311 methods such as the natural neighbor, nearest neighbor, and IDW interpolations. Since known

312 components of the scatter points are located in the two-dimensional X-Y plane, at least two

313 neighboring points are needed to form a tangent space for the manifold method. Therefore, for

314 simplicity, only two nearest neighbors are used in both manifold and IDW interpolation methods.

\section{2.3 Assimilating Meteorological Data for Improved Lake Circulation Modeling:}

$316 \quad$ Lake Michigan

317 The proposed method was first applied for the reconstruction of wind fields (time-varying 318 vector fields) over Lake Michigan. Hourly wind speed and direction data during April-

319 September 2008 were obtained from the National Data Buoy Center (NDBC) weather stations

320 surrounding the lake (Fig. 6). The wind measurements were adjusted to a $10 \mathrm{~m}$ anemometer

321 height using the profile methods described in Schwab (1987). Since the aerodynamic roughness

322 over the lake is much lower compared to its counterpart over the land, an empirical overland-

323 overlake adjustment was applied to the wind speeds recorded by overland stations (Schwab and

324 Beletsky, 1998). The datasets of wind speed and direction were converted to two coordinates in

325 the Cartesian coordinate system ( $x$ and $y$ directions).

326 Instead of using the cross-validation method to evaluate the interpolated wind data, results from

327 the hydrodynamic model of the lake were compared with current measurements to test the

328 applied method. To this end, a well-tested three-dimensional hydrodynamic model of the lake

329 (Safaie et al., 2016) was used. The model was based on the unstructured grid Finite Volume

330 Community Ocean Model (FVCOM; Chen et al., 2006) which was successfully used in the past

331 in ocean (Li et al., 2014), lake (Nguyen et al., 2014) and river (Anderson and Phanikumar, 2011) 
332 modeling. Details of the unstructured mesh used in the hydrodynamic model are presented in 333 Table 1.

334 Wind fields from April to September 2008 were reconstructed at the locations of nodes in the 335 numerical mesh. Other hourly meteorological observations related to heat flux fields, including 336 air temperature, cloud cover, dew point, long-wave solar radiation, short-wave solar radiation, 337 and relative humidity, obtained from the National Climatic Data Center (NCDC) and NDBC 338 stations, were interpolated over the computational grid using a smoothed natural neighbor 339 method with a smoothing radius of $30 \mathrm{~km}$. Air pressure was assumed to be constant $\left(10^{5} \mathrm{~Pa}\right)$ 340 through the course of the study and a constant startup water temperature with a value of $2.5^{\circ} \mathrm{C}$ 341 was used in the model. The overlake dew points were estimated from overland observations 342 using an empirical formula described in (Schwab and Beletsky, 1998). Air temperature and cloud 343 cover were used to estimate long-wave solar radiation (Parkinson and Washington, 1979) and 344 short-wave solar radiation was modeled using the clear-sky value and cloud cover (Nguyen et al., 345 2014). Six arc-second bathymetric data obtained from the NOAA National Geophysical Data 346 Center (NGDC) combined with two-meter resolution LIDAR data along the Indiana coast from 347 the National Oceanic and Atmospheric Administration (NOAA) were interpolated to the 348 numerical mesh using the natural neighbor method (Safaie et al., 2015).

349 Three bottom-mounted, upward-looking Acoustic Doppler Current Profilers (ADCPs) were 350 deployed at stations M, BB and S (Fig. 6) in southern Lake Michigan from early June to late 351 August 2008 to measure nearshore currents for model testing (Thupaki et al., 2013; Safaie et al., 352 2016). The hydrodynamic model was run from April to August 2008 to have a two-month spin353 up period. Evaluation of the manifold method was carried out by comparing the simulated 354 currents with data collected by the ADCPs. Comparisons between simulated and observed 
355 currents can be improved by identifying an optimal set of parameters in the manifold method.

356 These parameters include: an optimum number of the nearest neighbors to create a tangent space,

357 the time scale $c$, and parameters of the Gaussian kernel function. In addition, the method used for

358 creating a tangent space from a set of data points (Appendix-A) can be changed to improve the

359 agreement between simulated and observed currents. The manifold method for the reconstruction

360 of wind fields was directly applied to reconstruct the other six scalar observations to calculate the

361 heat flux fields. This time, however, $P^{v}$ is a scalar, rather than a vector.

\section{2.4 Assimilating Geophysical Data for Improved Lake Circulation Modeling: Gull}

$363 \quad$ Lake

364 In the second example, the bathymetry of Gull Lake was reconstructed using a manifold 365 method. The lake bathymetry data were collected using a SonTek RiverSurveyor M9 system. 366 The M9 system has an Acoustic Doppler Profiler (ADP) with two sets of four profiling beams 367 and one vertical acoustic beam (0.5-MHz echo-sounder) for river discharge measurements and 368 bathymetric surveys. The system was equipped with differential GPS with sub-meter precision 369 and mounted on a SonTeck hydroboard to avoid high pitch and roll angles. The vertical acoustic 370 beam has a range of $0.2 \mathrm{~m}$ to $80 \mathrm{~m}$ with an accuracy of $1 \%$ and a resolution of $0.001 \mathrm{~m}$. The 371 bathymetry survey was performed in four days (June 9 - June 12, 2015) by collecting data along

372 longitudinal and transverse transects of the lake with an approximate interval of $200 \mathrm{~m}$ between 373 each transect pair and sampling interval of $0.2 \mathrm{~m}-2 \mathrm{~m}$ along the transects depending on the boat 374 speed (Fig. 7).

375 In order to assimilate the bathymetry of the lake, a three-dimensional hydrodynamic model 376 based on FVCOM has been developed for the lake during the period of thermal stratification 
377 (June-August of 2014). The hydrodynamic equations were solved by the numerical model on an 378 unstructured grid and details are given in Table 1.

379 The meteorological observations for calculation of wind and heat flux fields were obtained 380 from NCDC, Weather Underground (https://www.wunderground.com), and the Kellogg 381 Biological Station Long-Term Ecological Research (KBS LTER, http://lter.kbs.msu.edu) 382 stations, a total of 22 locations surrounding Gull Lake from May to August (Fig. 8). Instead of a 383 constant air pressure, hourly air pressure data recorded by the KBS LTER stations were used to 384 improve the performance of the model. This also helped in the calculation of water density in 385 FVCOM based on a polynomial expression (Jackett and Mcdougall, 1995) that takes pressure 386 into account. After applying overland-overlake adjustments, all observations were interpolated 387 over the numerical mesh using a smoothed natural neighbor method with a smoothing radius of $38815 \mathrm{~km}$. This radius provided the best simulated results between the ranges of 0 to $30 \mathrm{~km}$. Air 389 temperatures were adjusted using the empirical formula of $T_{a}=0.4 T_{l a}+0.6 T_{w}$ (Schwab and 390 Beletsky, 1998), where $T_{a}$ is the adjusted air temperature over water, $T_{l a}$ is the air temperature 391 reported by overland stations, and $T_{w}$ is the surface water temperature. The surface water 392 temperature was collected using an Onset HOBO Pro v2 sensor with an accuracy of $0.2^{\circ} \mathrm{C}$. A 393 linearly varying startup water temperature was used with a value of $12^{\circ} \mathrm{C}$ at the water surface and $39444^{\circ} \mathrm{C}$ at the depth of $10 \mathrm{~m}$. The hydrodynamic model was tested using observed current data 395 measured using a Teledyne - RDI Sentinel-V ADCP (1000 kHz frequency with a bin size of 0.3 $396 \mathrm{~m}$ ) deployed in the nearshore waters of the lake in approximately $10 \mathrm{~m}$ of water (Fig. $7 \mathrm{~b}$ ). 397 Finally, the bathymetry of the lake interpolated to grid nodes using the manifold method was 398 assimilated into the model. 
413 in Michigan. The GR stretches 420 kilometers to the outlet at Grand Haven on Lake Michigan 414 and it is the longest river in Michigan. Shen et al. (2014) described the details of data input and 415 integration algorithms of PAWS+CLM; thus we simply introduce the basic data input and 416 processing information for our model in this section. We used a grid resolution of $1 \mathrm{~km} \mathrm{x} 1 \mathrm{~km}$ 417 for horizontal discretization which produced a $170 \times 195$ mesh for the GR watershed and 20 418 vertical layers to simulate the vadose zone dynamics by solving the Richards equation and 2 419

\subsection{Assimilating Precipitation Data for an Integrated, Distributed Hydrologic} model

In the third example, we simulate the hydrology of a watershed in the Great Lakes region using the spatial distribution of six-year (2000-2005) daily participation data over the Grand River (GR) watershed in Michigan using an integrated, process-based hydrologic model, PAWS+CLM (Shen and Phanikumar, 2010; Shen et al., 2013; Niu et al., 2014). The model is able to simulate different hydrologic components and states including channel discharge, surface runoff, evapotranspiration, groundwater, soil moisture, soil temperature, and vegetation growth. PAWS+CLM uses a structured finite-volume grid to solve the governing partial differential equations for different hydrologic components. Governing equations and numerical details of PAWS have been described in (Shen and Phanikumar, 2010) and in Table 2 of (Niu et al., 2014).

The Grand River (GR) watershed (Fig. 9) was selected as our study domain. GR watershed is located in the middle of Michigan's Lower Peninsula and it is the second largest watershed in Michigan. The watershed has a drainage area of $14,431 \mathrm{~km}^{2}$ and drains portions of 15 counties verical layers to simulate the vadose zone dynamics by solving the Richards equation and 2 layers for the groundwater domain (unconfined and confined aquifers) (Table 1). 
420 For topographic calculations (e.g. surface slope and overland flow), the $30 \mathrm{~m}$ resolution 421 National Elevation Dataset (NED, http:/ned.usgs.gov) from U.S. Geological Survey (USGS) was 422 used as the Digital Elevation Model (DEM). For river network simulation, National 423 Hydrography Dataset (NHD) from USGS was assimilated and reorganized as 'river segments' 424 with a length of one kilometer. We used the $30 \mathrm{~m}$ resolution raster data provided by the 425 Michigan Department of Natural Resources, i.e. the Integrated Forest Monitoring Assessment 426 and Prescription (IFMAP) dataset as the land use and land cover (LULC) layout (MDNR, 2010). 427 Soil information was obtained from Soil Survey Geographic (SSURGO) (Soil Survey Staff) 428 database from U.S. Department of Agriculture. This information was processed by the 429 pedotransfer functions provided in ROSETTA (Schaap et al., 2001) to provide soil properties of 430 water retention and unsaturated conductivities. Climate driven data (e.g. precipitation, daily 431 maximum temperature and minimum temperature, wind speed) are acquired as point input (Fig. 432 9) from National Climatic Data Center (NCDC, 2010) of the National Oceanic and Atmospheric 433 Administration (NOAA).

434 In this study, 14 rain gauges in the GR were selected to obtain the spatial distribution of rainfall 435 over the watershed and for assimilation into the model. Previous applications of the 436 PAWS+CLM model used the nearest neighbor method as the default for processing precipitation 437 data. In our work, the manifold method was tested by evaluating the stream discharge outputs of 438 PAWS+CLM against USGS data. The parameters of PAWS+CLM are listed in Table 1 of (Shen 439 et al., 2013), most of which are spatially distributed and have taken into account the spatial 440 heterogeneity of parameters such as the soil parameters and groundwater hydraulic 441 conductivities. 


\section{Results and Discussion}

\section{$443 \quad 3.1 \quad$ Analytical function}

444 True values of the analytical function at each of the randomly selected sampling locations were 445 compared with the estimated values obtained by the manifold method as well as other standard 446 interpolation methods. The performance statistics for this example are provided in Table 2. For

447 all methods, the approximation of the F7 function improved by increasing the number of 448 sampling points. In this particular example, the results show that the manifold method produced 449 better overall performance compared to the other three methods considered. However, the best 450 method in this example might perform differently on another test function or for a different 451 sampling point distribution; therefore, we examine the performance of the method for other 452 datasets in the following sections.

\section{$453 \quad 3.2$ Lake Michigan}

454 Due to the sparse distribution of weather stations around Lake Michigan, it was not clear $a$ 455 priori how many neighboring stations would provide an adequate representation of the data. 456 Since choosing a relatively few (e.g., three) neighboring stations in this situation would involve 457 using information from stations that are far apart as neighbors, we used kernel regression to 458 assign weights to each station depending on the distance from the point of interest. For each 459 node of the numerical grid of Lake Michigan, $k$ number of nearest neighbors were selected and 460 their assigned weights were projected to a low-dimensional subspace. The free parameters in the 461 method are $c$ (time scale), $\sigma$ (the parameter used in kernel regression), and $k$. The standard 462 deviation of weather station distances from the point of interest was used for the parameter $\sigma$ in 463 kernel regression. Performance of the manifold method as measured by a comparison of 
464 simulated and observed currents in Lake Michigan is summarized in Table 3 relative to the other 465 standard methods considered. We note that the manifold method based on kernel weighting 466 considering all stations produced the best overall performance as measured by the root mean 467 squared error (RMSE) between the observed and simulated currents. The performance of the 468 method without kernel regression and with only three neighboring stations was comparable to 469 the other methods but slightly inferior to the natural and nearest neighbor methods. Fig. 10 470 shows the RMSE and $\mathrm{R}^{2}$ values for different numbers of nearest neighbors at different ADCP 471 locations. Having all stations to create the tangent space for the manifold method resulted in a 472 better representation of wind fields, and improved the results of the hydrodynamic model (Fig. 47311 shows the comparison at station M). We can see that the manifold method performs better 474 than the IDW method at two of the offshore stations ( $\mathrm{M}$ and BB) but not at the nearshore 475 location $\mathrm{S}$. We believe that the reason for this has to do with the fact that in the nearshore region 476 there are a number of additional processes (waves, wave-current interactions etc) which are not 477 simulated in our model. Therefore model performance in that region cannot be directly attributed 478 to the wind field. At the other two offshore stations M and BB, where the flow is predominantly 479 wind-driven, an improvement in the simulated hydrodynamic fields can be seen.

480 Finally, cross-validation was used to compare the performance of the manifold method with 481 other standard methods for the same Lake Michigan datasets. The performance metrics are 482 summarized in Table 4. In this cross-validation method, one weather station was withheld to be 483 used later for validating the manifold method, and all other stations surrounding the lake were 484 used for the manifold training set. This process was repeated so that each weather station was 485 given a chance to be part of this validation process. Based on these results the proposed manifold 486 method with three nearest neighbors gave better results compared to other standard methods. 
487 However, the performance of the hydrodynamic model based on these methods was relatively 488 inferior compared to the performance of the model when the manifold method used all 489 neighboring points. All different versions of the manifold methods had reasonable computational 490 efficiency. The computational time for the O-kriging was high due to the time needed for finding 491 the best variogram at each time step.

\section{$492 \quad 3.3$ Gull Lake}

493 Wind and heat flux fields of the Gull Lake were interpolated over the numerical mesh of the 494 lake using the natural neighbor method. Then, the bathymetry of the lake was interpolated over 495 the mesh using the same natural neighbor method to develop the initial version of the lake 496 hydrodynamic model. The raw bathymetry data, which has some regions of steep bathymetry 497 change, created artificial currents in the model due to an error in the pressure gradient force 498 introduced by the sigma-coordinate system of FVCOM (Mellor et al., 1998). Therefore, the 499 interpolated bathymetry was smoothed with a radius of $100 \mathrm{~m}$ in order to reduce the errors. The 500 results of the developed model using natural neighbor method and IDW with three nearest 501 neighbors are presented in Fig. 12.

502 The model was used to assimilate the bathymetry of the lake based on the manifold method.

503 The bathymetry data were reconstructed from the tangent space of the manifold with three 504 nearest neighbors and smoothed with the same method described above. The hydrodynamic 505 model was run with the reconstructed bathymetry. The final comparisons of the vertically506 averaged velocity profiles at the ADCP location are presented in Fig. 12. The best value of $\sigma$ 507 used in kernel regression was equal to the standard deviation of distances of observational points 508 where water depth values are available within a search radius of $50 \mathrm{~m}$ from the point of interest. 
509 When the number of samples within this radius was smaller than $100, \sigma$ value was calculated

510 based on locations of 100 nearest samples. This method is more accurate when enough samples

511 are available around an estimated point, unless selecting 100 samples itself does a reasonable

512 job. RMSE values (m/s) of eastward and northward velocities in Gull Lake for comparison of the

513 manifold method with other standard methods used in limnology and oceanography are

514 presented in Table 5. The statistics of cross-validation for all (=71) measured longitudinal and

515 transverse transects are shown in Table 6. The cross-validation was performed by omitting one

516 transect at each step and calculating the bathymetry for that transect from the rest of the

517 observation data and repeating the process for all other transects.

\section{$518 \quad 3.4$ Grand River watershed}

519 The daily distributions of precipitation over the GR watershed for the period 2000 to 2005 were

520 reconstructed from selected rain gauges using the manifold method. The precipitation fields over

521 a period of six years were tested by comparing the simulated and observed stream discharges at

522 selected USGS gaging stations within the watershed. In this example, kernel regression with a

523 standard deviation of all rain gauge distances from the estimated points was used in the manifold

524 method. The tangent space at each grid point of the numerical model was obtained from three-

525 nearest neighbors around that point. The PAWS+CLM model was run with precipitation

526 distributions built using the manifold, natural neighbor, nearest neighbor, and inverse distance

527 methods. . The final comparisons of simulated and observed stream flows for USGS gauges are

528 presented in Figs. 13 and 14. Model performance metrics (NASH, Absolute Percent Bias (APB), and

529 RMSE) were computed to evaluate the performance of the manifold method with other standard methods

530 (Table 7). The manifold method provided superior results as can be seen from the improved

531 representation of baseflow over the simulation period. This can be seen clearly from semi-log 
532 plots of the stream hydrograph comparison. The cross-validation results for precipitation are

533 presented in Table 8. In this method, data from one rain gauge was withheld and data from the

534 rest of the gauges were used for manifold training. This process was repeated for all 14 rain

535 gauges. Since rain gauges have a sparse distribution, the cross-validation method could not

536 identify the best method for this case. The results of the integrated, distributed hydrologic model,

537 however, clearly demonstrate the efficacy of the manifold method in the reconstruction of

538 precipitation fields. In particular, the better simulated baseflow shows the strength of using

539 precipitation data based on the manifold method to improve the simulation of heterogeneous

540 partitioning of surface runoff and infiltration processes.

\section{Conclusions}

542 We presented a novel manifold method of reconstructing spatio-temporal data for assimilating 543 geophysical and meteorological data in integrated land surface subsurface, and oceanic/lake

544 models. All three case studies illustrate the superior performance of the presented manifold

545 algorithm over standard methods in terms of accuracy and computational efficiency. The

546 hydrodynamic model of Lake Michigan based on the manifold method of reconstructing wind

547 fields produced better performance relative to the other methods. The best results were obtained

548 using kernel regression applied to all weather stations (neighbors). However, the cross-validation

549 results show that the results of the three nearest neighbors were better than the other methods.

550 The Gull Lake model results indicated that the proposed method has the ability to reconstruct 551 geophysical data at unsampled locations. The use of spatiotemporal precipitation fields 552 constructed using the manifold method produced better stream discharge simulations compared 553 to similar results from the nearest neighbor, natural neighbor and IDW interpolation methods in a 
554 large watershed $\left(>10000 \mathrm{~km}^{2}\right)$. Finally, all three examples show that evaluating the performance

555 of interpolation methods using the cross-validation method without considering the data structure

556 and the purpose of interpolation can lead to misleading conclusions about the relative

557 performance of the methods considered. The comparisons presented here indicate that manifold 558 methods show promise for modeling Earth system processes based on data from sensor

559 networks. Our future work will combine manifold methods with approaches such as the EnKF

560 method to further improve process-based modeling of land surface, subsurface and lake/ocean

561 models.

562 Based on the results presented, we note that: (1) Details of the manifold method such as the 563 tangent space estimation, the distance metric that defines spatiotemporal proximity and other

564 details can be further improved to improve the performance of the manifold method; however,

565 these topics are beyond the scope of the present paper. (2) We do not claim that the manifold

566 method provides superior performance on all datasets and for all performance metrics but from

567 the examples considered here it appears that the manifold method may offer an attractive method

568 that is comparable or superior to other standard methods. More research is needed to understand

569 the relative strengths and weaknesses of different manifold-based approaches compared to 570 standard methods.

\section{Acknowledgments}

572 This work was supported by a grant from the National Science Foundation, CyberSEES program 573 (Award \# 1331852). We thank Elena Litchman, Pam Woodruff, Jim Allen, Mike Gallagher, 574 Andrew Fogiel, and Tuan D. Nguyen for their assistance with field data collection in Gull Lake. 
575 We gratefully acknowledge the use of data from the Kellogg Biological Station LTER which is

576 supported by NSF (DEB 1027253) and by Michigan State University AgBioResearch.

\section{Appendix-A. Tangent Space Estimation}

578 To understand the local geometry of the surface $f(x)$ near a point $x \in \square^{n}$, we consider the first-

579 order Taylor series expansion of the surface:

580

$f(\bar{x})=f(x)+\frac{\partial f(x)}{\partial x}(\bar{x}-x)+O\left(\|\bar{x}-x\|^{2}\right)=f(x)+J_{f}(\bar{x}-x)+O\left(\|\bar{x}-x\|^{2}\right)$

581 where $J_{f}(x) \in \square^{D \times n}$ is the Jacobian matrix of $f$ at the point $x$. If the components of $f(x)$ can

582 be written as: $f(x)=\left[f_{1}(x), f_{2}(x), f_{3}(x) \cdots f_{D}(x)\right]^{T}$ and $x=\left[x_{1}, x_{2}, x_{3} \cdots x_{n}\right]^{T}$, then the Jacobian

583 can be written as:

$584 \quad J_{f}(x)=\left[\begin{array}{ccc}\frac{\partial f_{1}}{\partial x_{1}} & \cdots & \frac{\partial f_{1}}{\partial x_{n}} \\ \vdots & \vdots & \vdots \\ \frac{\partial f_{D}}{\partial x_{1}} & \cdots & \frac{\partial f_{D}}{\partial x_{n}}\end{array}\right]$

585 To understand the local shape of the surface in equation (A1), we seek to determine the space

$586(\bar{x}-x)$, such that as we move away from $x$, the value of the function doesn't change to within

587 first order. This is equivalent to finding the space $T$ such that:

$588 T=\left\{(\bar{x}-x) \mid J_{f}(x)(\bar{x}-x)=0\right\}$ 
589 This space is the tangent space to the surface at point $x$ and is the right null space of the Jacobian

590 matrix $J_{f}(x)$. The space orthogonal to the tangent space is the row space of the Jacobian and

591 orthogonal representations of these spaces can be obtained from SVD. The right null space of

$592 J_{f}$ is the columns of $V$ corresponding to zero singular values. Therefore, the tangent space of

593 the manifold $M$ at $y=f(x)$ is:

$594 T(M)=\operatorname{span}\left(J_{f}(x)\right)$

595 From a practical computation point of view, given a set of sample points $y=\left\{y_{1}, y_{2}, y_{3}, \cdots y_{m}\right\}$, a

596 simple method of constructing the tangent space is to approximate it as the line/surface obtained

597 by joining the local neighboring points. The tangent space can also be directly estimated using

598 SVD. If $C^{m}$ denotes the local covariance matrix:

$599 \quad C^{m}=\frac{1}{m} \sum_{i=1}^{m} y_{i} y_{i}^{T}=U \Sigma U^{T}$

600 where $U=\left[u_{1}, u_{2}, u_{3}, \cdots u_{D}\right]$ and $\Sigma=\operatorname{diag}\left[\lambda_{1}, \lambda_{2}, \lambda_{3} \cdots \lambda_{D}\right]$ denote the eigenvector and eigenvalue

601 matrices respectively, then the optimal (in a least-squares sense) $n$-dimensional linear subspace is

602 the span of the $n$-largest eigenvectors in $U$ :

$603 T(M) \cong \operatorname{span}\left\{u_{1}, u_{2}, u_{3}, \cdots u_{n}\right\}$

\section{5. References}

605 Allard, W.K., Chen, G., Maggioni, M., 2012. Multi-scale geometric methods for data sets II:

606 Geometric Multi-Resolution Analysis. Appl. Comput. Harmon. Anal. 32, 435-462.

607 doi:10.1016/j.acha.2011.08.001 
Anderson, E.J., Phanikumar, M.S., 2011. Surface storage dynamics in large rivers: Comparing three-dimensional particle transport, one-dimensional fractional derivative, and multirate transient storage models. Water Resources Research 47, 1-15. doi:10.1029/2010WR010228

611 Anquetin, S., Braud, I., Vannier, O., Viallet, P., Boudevillain, B., Creutin, J.-D., Manus, C., 612 2010. Sensitivity of the hydrological response to the variability of rainfall fields and soils for the 613 Gard 2002 flash-flood event. J. Hydrol. 394, 134-147. doi:10.1016/j.jhydrol.2010.07.002

614 Barth, A., Azcárate, A. A., Joassin, P., Jean-Marie, B., Troupin, C., 2008. Introduction to 615 Optimal Interpolation and Variational Analysis. Presented at the SESAME Summer School, 616 SESAME Summer School, Varna, Bulgaria.

617 Belkin, M., Niyogi, P., 2003. Laplacian eigenmaps for dimensionality. Speech Commun. 1, 349618367.

619 Bell, V.A., Moore, R.J., 2000. The sensitivity of catchment runoff models to rainfall data at 620 different spatial scales. Hydrol. Earth Syst. Sci. 4, 653-667. doi:10.5194/hess-4-653-2000

621 Beven, K.J., Hornberger, G.M., 1982. Assessing the Effect of Spatial Pattern of Precipitation in 622 Modeling Stream Flow Hydrographs. J. Am. Water Resour. Assoc. 18, 823-829.

623 doi:10.1111/j.1752-1688.1982.tb00078.x

624 Blumberg, A.F., Mellor, G.L., 1987. A description of a three-dimensional coastal ocean 625 circulation model. Am. Geophys. Union 1-16.

626 Candès, E.J., Li, X., Ma, Y., Wright, J., 2011. Robust principal component analysis? J. ACM 58, 627 1-37. doi:10.1145/1970392.1970395

628 Carin, L., Baraniuk, R.G., Cevher, V., Dunson, D., Jordan, M.I., Sapiro, G., Wakin, M.B., 2011. 629 Learning Low-Dimensional Signal Models. IEEE Signal Process. Mag. 28.

630 doi:10.1109/MSP.2010.939733

631 Chen, C., Beardsley, R., Cowles, G., 2006. An Unstructured Grid, Finite-Volume Coastal Ocean 632 Model (FVCOM) System. Oceanography 19, 78-89. doi:10.5670/oceanog.2006.92

633 Daly, C., Gibson, W.P., Taylor, G.H., Johnson, G.L., Pasteris, P., 2002. A knowledge-based 634 approach to the statistical mapping of climate. Clim. Res. 22, 99-113.

635 Dang, C., Aghagolzadeh, M., Radha, H., 2014. Image Super-Resolution via Local Self-Learning 636 Manifold Approximation. IEEE Signal Process. Lett. 21, 1245-1249.

637 doi:10.1109/LSP.2014.2332118

638 Dang, C., Radha, H., 2015. Fast Image Super Resolution via Selective Manifold Learning of 639 High Resolution Patches. Presented at the IEEE Proceedings of International Conference on 640 Image Processing (ICIP15), IEEE Proceedings of International Conference on Image Processing 641 (ICIP15), Québec City, Canada. 
Dang, C.T., Aghagolzadeh, M., Moghadam, A.A., Radha, H., 2013. Single image super

643 resolution via manifold linear approximation using sparse subspace clustering, in: Global

644 Conference on Signal and Information Processing (GlobalSIP), 2013 IEEE. IEEE, Austin, Texas,

645 U.S.A., pp. 949-952.

646 Evensen, G., 2006, Data Assimilation: The Ensemble Kalman Filter, Springer, N. Y.

647 Golub, G.., Van Loan, C.F., 2013. Matrix Computations, 4th ed. Baltimore: Johns Hopkins, pp. 648756.

649 Haidvogel, D.B., Beckmann, A., 1999. Numerical Ocean Circulation Modeling. Imp. Coll. Press.

650 Zhang, H., Mendoza-Sanchez, I., Miller, E.L., Abriola, L.M., 2016. Manifold Regression

651 Framework for Characterizing Source Zone Architecture. IEEE Transactions on Geoscience and

652 Remote Sensing 54, 3-17. doi:10.1109/TGRS.2015.2448086

653 Jackett, D.R., Mcdougall, T.J., 1995. Minimal Adjustment of Hydrographic Profiles to Achieve

654 Static Stability. J. Atmospheric Ocean. Technol. 12, 381-389. doi:10.1175/1520-

655 0426(1995)012<0381:MAOHPT >2.0.CO;2

656 Lazzaro, D., Montefusco, L.B., 2002. Radial basis functions for the multivariate interpolation of 657 large scattered data sets. J. Comput. Appl. Math., Int. Congress on Computational and Applied 658 Mathematics 2000 140, 521-536. doi:10.1016/S0377-0427(01)00485-X

659 Li, J., Heap, A.D., 2008. A review of spatial interpolation methods for environmental scientists.

660 Geoscience Australia, Canberra.

661 Li, L., Losser, T., Yorke, C., Piltner, R., 2014. Fast Inverse Distance Weighting-Based 662 Spatiotemporal Interpolation: A Web-Based Application of Interpolating Daily Fine Particulate 663 Matter PM2:5 in the Contiguous U.S. Using Parallel Programming and k-d Tree. Int. J. Environ. 664 Res. Public. Health 11, 9101-9141. doi:10.3390/ijerph110909101

665 Li, L., Revesz, P., 2004. Interpolation methods for spatio-temporal geographic data. Comput. 666 Environ. Urban Syst. 28, 201-227. doi:10.1016/S0198-9715(03)00018-8

667 Li, R. et al., 2014. Observed wintertime tidal and subtidal currents over the continental shelf in 668 the northern South China Sea, J. Geophys. Res. Oceans, 119(8), pp. 5289-5310, doi:10.1002/ 669 2014JC009931.

670 Luo, W., Taylor, M.C., Parker, S.R., 2008. A comparison of spatial interpolation methods to 671 estimate continuous wind speed surfaces using irregularly distributed data from England and 672 Wales. Int. J. Climatol. 28, 947-959. doi:10.1002/joc.1583

673 Ly, S., Charles, C., Degré, A., 2011. Geostatistical interpolation of daily rainfall at catchment 674 scale: the use of several variogram models in the Ourthe and Ambleve catchments, Belgium.

675 Hydrol. Earth Syst. Sci. 15, 2259-2274. doi:10.5194/hess-15-2259-2011 
MacEachren, A.M., Davidson, J.V., 1987. Sampling and isometric mapping of continuous geographic surfaces. Am. Cartogr. 14, 299-320.

Masih, I., Maskey, S., Uhlenbrook, S., Smakhtin, V., 2011. Assessing the Impact of Areal Precipitation Input on Streamflow Simulations Using the SWAT Model1. JAWRA J. Am. Water Resour. Assoc. 47, 179-195. doi:10.1111/j.1752-1688.2010.00502.x

Ma, Y., Niyogi, P., Sapiro, G., Vidal, R., 2011. Dimensionality reduction via subspace and submanifold learning. IEEE Signal Process. Mag. 28, 14-126. doi:10.1109/MSP.2010.940005

Mellor, G.L., Oey, L.Y., Ezer, T., 1998. Sigma coordinate pressure gradient errors and the seamount problem. Journal of Atmospheric and Oceanic Technology 15, 1122-1131.

Merwade, V., 2009. Effect of spatial trends on interpolation of river bathymetry. J. Hydrol. 371, 169-181. doi:10.1016/j.jhydrol.2009.03.026

Mordohai, P., Medioni, G., 2010. Dimensionality estimation, manifold learning and function approximation using tensor voting. J. Mach. Learn. Res. 11, 411-450.

Moradkhani, H., Sorooshian, S., Gupta, H.V., Houser, P.R., 2005. Dual state-parameter estimation of hydrological models using ensemble Kalman filter, Adv. Water Resour. 28, 135$691 \quad 147.4$

692 Nguyen, T.D., Thupaki, P., Anderson, E.J., Phanikumar, M.S., 2014. Summer circulation and 693 exchange in the Saginaw Bay-Lake Huron system. J. Geophys. Res. Oceans 119, 2713-2734. 694 doi:10.1002/2014JC009828

695 Nicótina, L., Alessi Celegon, E., Rinaldo, A., Marani, M., 2008. On the impact of rainfall patterns on the hydrologic response. Water Resour. Res. 44, 1-14. doi:10.1029/2007WR006654

Niu, J., Phanikumar, M.S., 2015. Modeling watershed-scale solute transport using an integrated, process-based hydrologic model with applications to bacterial fate and transport. J. Hydrol. 529, 35-48. doi:10.1016/j.jhydrol.2015.07.013

Niu, J., Shen, C., Li, S.-G., Phanikumar, M.S., 2014. Quantifying storage changes in regional MODIS products. Water Resour. Res. 50, 7359-7377. doi:10.1002/2014WR015589

Obled, C., Wendling, J., Beven, K., 1994. The sensitivity of hydrological models to spatial rainfall patterns: an evaluation using observed data. J. Hydrol. 159, 305-333. doi:10.1016/00221694(94)90263-1

Oleson, K.W., Lawrence, D.M., Gordon, B., Flanner, M.G., Kluzek, E., Peter, J., Levis, S., Swenson, S.C., Thornton, E., Feddema, J., others, 2010. Technical description of version 4.0 of the Community Land Model (CLM) (No. NCAR/TN-478+STR), NCAR Technical Note. National Center for Atmospheric Research, Boulder, Colorado. 
710 Parkinson, C.L., Washington, W.M., 1979. A large-scale numerical model of sea ice. J. Geophys. 711 Res. Oceans 84, 311-337. doi:10.1029/JC084iC01p00311

712 Pathiraja, S., Marshall, L., Sharma, A., Moradkhani, H., 2016, Hydrologic modeling in dynamic 713 catchments: A data assimilation approach, Water Resour. Res. 52, 1-23.

714 doi:10.1002/2015WR01719

715 Press, W.H., Flannery, B.P., Teukolsky, S.A., Vetterling, W.T., 2007. Numerical Recipes in

716 C++: The Art of Scientific Computing, third ed. Cambridge University Press, New York.

717 Renka, R.J., Brown, R., 1999. Algorithm 792: Accuracy Test of ACM Algorithms for

718 Interpolation of Scattered Data in the Plane. ACM Trans Math Softw 25, 78-94.

719 doi:10.1145/305658.305745

720 Roweis, S.T., 2000. Nonlinear dimensionality reduction by locally linear embedding. Science 721 290, 2323-2326. doi:10.1126/science.290.5500.2323

722 Safaie, A., Wendzel, A., Ge, Z., Nevers, M.B., Whitman, R.L., Corsi, S.R., Phanikumar, M.S., 723 2016. Comparative Evaluation of Statistical and Mechanistic Models of Escherichia coli at 724 Beaches in Southern Lake Michigan. Environ. Sci. Technol., 50, 2442-2449.

725 doi:10.1021/acs.est.5b05378

726

727

728

729

730

732

733

734
Schaap, M.G., Leij, F.J., van Genuchten, M.T., 2001. rosetta: a computer program for estimating soil hydraulic parameters with hierarchical pedotransfer functions. J. Hydrol. 251, 163-176. doi:10.1016/S0022-1694(01)00466-8

Schuurmans, J.M., Bierkens, M.F.P., 2007. Effect of spatial distribution of daily rainfall on interior catchment response of a distributed hydrological model. Hydrol. Earth Syst. Sci. Discuss. 11, 677-693.

Schwab, D.J., 1987. Simulation and forecasting of Lake Erie storm surges. Mon. Weather Rev. 106, 1476-1487.

Schwab, D.J., Beletsky, D., 1998. Lake Michigan Mass Balance Study: Hydrodynamic modeling project (No. NOAA Technical Memorandum ERL GLERL-108). Great Lakes Environmental Research Laboratory, Ann Arbor, MI.

Shen, C., Niu, J., Fang, K., 2014. Quantifying the effects of data integration algorithms on the outcomes of a subsurface-land surface processes model. Environ. Model. Softw. 59, 146-161. doi:10.1016/j.envsoft.2014.05.006

Shen, C., Niu, J., Phanikumar, M.S., 2013. Evaluating controls on coupled hydrologic and vegetation dynamics in a humid continental climate watershed using a subsurface-land surface processes model. Water Resour. Res. 49, 2552-2572. doi:10.1002/wrcr.20189 
Shen, C., Phanikumar, M.S., 2010. A process-based, distributed hydrologic model based on a large-scale method for surface-subsurface coupling. Adv. Water Resour. 33, 1524-1541. doi:10.1016/j.advwatres.2010.09.002

Šiljeg, A., Lozić, S., Šiljeg, S., 2015. A comparison of interpolation methods on the basis of data obtained from a bathymetric survey of Lake Vrana, Croatia. Hydrol. Earth Syst. Sci. 19, 36533666. doi:10.5194/hess-19-3653-2015

Sun, X., Mein, R.G., Keenan, T.D., Elliott, J.F., 2000. Flood estimation using radar and raingauge data. J. Hydrol. 239, 4-18. doi:10.1016/S0022-1694(00)00350-4

Suparta, W., Rahman, R., 2016. Spatial interpolation of GPS PWV and meteorological variables over the west coast of Peninsular Malaysia during 2013 Klang Valley Flash Flood. Atmospheric Res. 168, 205-219. doi:10.1016/j.atmosres.2015.09.023

Syed, K.H., Goodrich, D.C., Myers, D.E., Sorooshian, S., 2003. Spatial characteristics of thunderstorm rainfall fields and their relation to runoff. J. Hydrol. 271, 1-21. doi:10.1016/S00221694(02)00311-6

Tenenbaum, J.B., De Silva, V., Langford, J.C., 2000. A global geometric framework for nonlinear dimensionality reduction. Science 290, 2319-2323.

Tetzlaff, D., Uhlenbrook, S., 2005. Significance of spatial variability in precipitation for processoriented modelling: results from two nested catchments using radar and ground station data. Hydrol. Earth Syst. Sci. 9, 29-41. doi:10.5194/hess-9-29-2005

Thupaki, P., M.S. Phanikumar and R.L. Whitman, 2013. Solute dispersion in the coastal boundary layer of southern Lake Michigan, J. Geophys. Res. Oceans, vol. 118, No. 3, pp. 16061617, doi: 10.1002 / jgrc.20136 (2013)

Thupaki, P., Phanikumar, M.S., Schwab, D.J., Nevers, M.B., Whitman, R.L., 2013. Evaluating the role of sediment-bacteria interactions on Escherichia coli concentrations at beaches in southern Lake Michigan., J. Geophys. Res. Oceans118, 7049-7065. doi:10.1002/2013JC008919

van der Maaten, L.J., Postma, E.O., van den Herik, H.J., 2009. Dimensionality reduction: A comparative review. J. Mach. Learn. Res. 10, 66-71.

Victor, G., Pollack, A., 2010. Differential topology. American Mathematical Soc.

Wendzel, A., 2014. Constraining mechanistic models of indicator bacteria at recreational beaches in Lake Michigan using easily-measurable environmental variables (M. Sc. Dissertation). Michigan State University, East Lansing. MI.

Xue, P., Schwab, D.J., Hu, S., 2015. An investigation of the thermal response to meteorological forcing in a hydrodynamic model of Lake Superior. J. Geophys. Res. Oceans 120, 5233-5253. doi:10.1002/2015JC010740 
778 Yan, Y., Xiao, F., Du, Y., 2014. Construction of lake bathymetry from MODIS satellite data and

779 GIS from 2003 to 2011. Chin. J. Oceanol. Limnol. 32, 720-731. doi:10.1007/s00343-014-3185-4

780 Yang, K., Watanabe, T., Koike, T., Li, X., Fujii, H., Tamagawa, K., Ma, Y., Ishikawa, H., 2007.

781 Auto-calibration system developed to assimilate AMSR-E data into a land surface model for

782 estimating soil moisture and the surface energy budget. J. Meteorol. Soc. Jpn. 85A, 229-242.

783

784

785

786

787

788

789

$790 \quad$ List of Figures

791 Fig. 1. Some examples of manifolds (a) torus, (b) sphere, and (c) a two-dimensional cross-section of a

792 six-dimensional Calabi-Yau manifold.

793 Fig. 2. Illustration of the proposed manifold approach for estimation of missing data at point $P_{0}$.

794 Fig. 3. A tangent space created from the set of nearest points using (a) coordinates of selected

795 neighborhoods or (b) Kernel regression.

796 Fig. 4. Manifolds representing (a) bathymetry of Gull Lake and (b) wind components over Lake

797 Michigan in three dimensional space.

798 Fig. 5. (a) Analytical function used to test the manifold method for interpolation of scattered data.

799 Random sampling was used to generate scatter points as shown in figures (b, 30 points), (c, 60 points) and

800 (d, 90 points) to reconstruct the function. 
Fig. 6. Locations of the ADCPs deployed during summer 2008 and weather stations surrounding Lake Michigan.

803 Fig. 7(a). Bathymetry of Gull Lake. (b) Boat tracks generated during the sampling survey in Gull Lake.

$804 \quad$ Fig. 8. Selected weather stations surrounding Gull Lake.

805 Fig. 9. Map of Grand River watershed showing the locations of the USGS gauges, rain gauges and

806 National Hydrography Dataset (NHD) streams.

807 Fig. 10. Performance of the manifold method evaluated using observed and simulated currents at different stations in Lake Michigan. Different number of nearest neighbors were used to reconstruct the wind field using the manifold method with kernel regression.

810 Fig. 11. Comparison of simulated (black lines) and observed (red lines) vertically averaged currents at the location M in Lake Michigan. (a) Alongshore velocity (b) Cross-shore velocity

812 Fig. 12. Comparison of simulated (black lines) and observed (red lines) vertically averaged currents at the 813 ADCP location in Gull Lake. (a) Eastward velocity and (b) Northward velocity

814 Fig. 13. Comparison of simulated and observed stream flows for USGS gauge \#04116000 in (a) a linear 815 scale, and (b) logarithmic scale.

816 Fig. 14. Comparison of simulated and observed stream flows for USGS gauge \#04119000 in (a) a linear 817 scale, and (b) logarithmic scale.

\section{List of Tables}

Table 1. Properties of the numerical grids used for the hydrodynamic and hydrologic

\begin{tabular}{lcccccc}
\hline Model & $\begin{array}{c}\text { Grid } \\
\text { Classification }\end{array}$ & $\begin{array}{c}\text { Element } \\
\text { shape }\end{array}$ & $\begin{array}{c}\text { Grid } \\
\text { Resolution }\end{array}$ & \# Nodes & \# Elements & $\begin{array}{c}\text { \#Vertical } \\
\text { layers }\end{array}$ \\
\hline FVCOM (Lake Michigan) & Unstructured & Triangle & $4 \mathrm{~m}-5 \mathrm{~km}$ & 12,684 & 23,602 & 20 \\
\hline FVCOM (Gull Lake) & Unstructured & Triangle & $8-100 \mathrm{~m}$ & 5,132 & 9,361 & 20 \\
\hline PAWS (Grand River) & Structured & Quadrilateral & $1 \mathrm{~km}$ & 33,150 & 32,786 & 22 \\
\hline
\end{tabular}

Table 2. Cross-validation results for the analytical function based on different sampling points selected randomly. 


\begin{tabular}{clcccccc}
\hline $\begin{array}{c}\text { Sample } \\
\text { size }\end{array}$ & \multicolumn{1}{c}{ Method } & $\mathrm{R}^{2}$ & RMSE & Fn & PBIAS & NASH & APB (\%) \\
\hline \multirow{3}{*}{30} & Manifold & 0.667 & 0.778 & 0.710 & 14.420 & 0.416 & 0.652 \\
& Natural neighbor & 0.582 & 0.876 & 0.799 & -8.866 & 0.259 & 0.713 \\
& Nearest neighbor & 0.619 & 0.882 & 0.804 & -14.689 & 0.250 & 0.669 \\
& IDW & 0.577 & 0.870 & 0.793 & 35.847 & 0.270 & 0.727 \\
& & & & & & & \\
& Manifold & 0.846 & 0.579 & 0.531 & -33.924 & 0.703 & 0.472 \\
& Natural neighbor & 0.816 & 0.615 & 0.564 & 16.912 & 0.664 & 0.466 \\
& Nearest neighbor & 0.779 & 0.720 & 0.660 & -34.524 & 0.540 & 0.512 \\
& IDW & 0.832 & 0.603 & 0.553 & -44.292 & 0.677 & 0.469 \\
& & & & & & & \\
& Manifold & 0.891 & 0.502 & 0.432 & -14.103 & 0.791 & 0.400 \\
& Natural neighbor & 0.874 & 0.539 & 0.464 & -10.666 & 0.759 & 0.344 \\
& Nearest neighbor & 0.867 & 0.571 & 0.491 & -28.777 & 0.730 & 0.446 \\
& IDW & 0.859 & 0.567 & 0.487 & -5.974 & 0.735 & 0.416 \\
\hline
\end{tabular}

826

827

828

829

830 Table 3. RMSE values $(\mathrm{m} / \mathrm{s})$ of alongshore and cross-shore velocities for comparison of the manifold 831 method with other standard methods used in limnology and oceanography

\begin{tabular}{lccccccccc}
\hline \multirow{2}{*}{ Method } & \multicolumn{2}{c}{ Loaction: $\mathrm{M}$} & & \multicolumn{2}{c}{ Location: BB } & & \multicolumn{2}{c}{ Location: $\mathrm{S}$} \\
\cline { 2 - 3 } O-kriging & RMSE u & RMSE v & & RMSE u & RMSE v & & RMSE u & RMSE v \\
Nearest Neighbor & 0.0385 & 0.0290 & & 0.0590 & 0.0349 & & 0.0540 & 0.0152 \\
Natural Neighbor & 0.0363 & 0.0286 & & 0.0580 & 0.0348 & & 0.0545 & 0.0152 \\
Manifold (3 NBR) & 0.0366 & 0.0275 & & 0.0553 & 0.0334 & & 0.0515 & 0.0158 \\
Manifold+Kernel (3 NBR) & 0.0383 & 0.0276 & & 0.0594 & 0.0346 & & 0.0568 & 0.0158 \\
Manifold+Kernel (all NBR) & 0.0304 & 0.0265 & & 0.0531 & 0.0312 & & 0.0568 & 0.0154 \\
IDW (all NBR) & 0.0328 & 0.0267 & & 0.0535 & 0.0316 & & 0.0498 & 0.0155 \\
\hline
\end{tabular}


Table 4. Cross-validation results for wind field over Lake Michigan.

\begin{tabular}{lccccc}
\hline Method & $\mathrm{R}^{2} \mathrm{u}$ & $\mathrm{R}^{2} \mathrm{v}$ & $\mathrm{RMSEu}$ & $\mathrm{RMSEv}$ & Computational time (s) \\
\hline O-kriging & 0.441 & 0.572 & 3.497 & 3.853 & 92463.8 \\
Nearest Neighbor & 0.666 & 0.743 & 2.792 & 3.044 & 18.6 \\
Natural Neighbor & 0.693 & 0.794 & 2.558 & 2.750 & 183.6 \\
Manifold (3 NBR) & 0.690 & 0.801 & 2.433 & 2.595 & 28.1 \\
Manifold+Kernel (3 NBR) & 0.710 & 0.806 & 2.392 & 2.566 & 55.1 \\
Manifold+Kernel (all NBR) & 0.547 & 0.681 & 2.884 & 3.129 & 77.3 \\
IDW (3 NBR) & 0.724 & 0.822 & 2.278 & 2.458 & 69.3 \\
\hline
\end{tabular}

Table 5. RMSE values ( $\mathrm{m} / \mathrm{s}$ ) of eastward and northward velocities in Gull Lake for comparison of the manifold method with other standard methods used in limnology and oceanography

\begin{tabular}{lcc}
\hline Method & RMSE u & RMSE v \\
\hline Natural Neighbor & 0.0090 & 0.0205 \\
Manifold+Kernel (3 NBR) & 0.0098 & 0.0200 \\
IDW (3 NBR) & 0.0095 & 0.0204 \\
\hline
\end{tabular}

Table 6. Cross-validation results for Gull Lake bathymetry.

\begin{tabular}{lccccc}
\hline Method & $\mathrm{R}^{2}$ & RMSE $(\mathrm{m})$ & Fn & NASH & PBIAS \\
\hline Manifold & 0.890 & 2.011 & 0.222 & 0.678 & -14.016 \\
Natural Neighbor & 0.925 & 1.288 & 0.170 & 0.468 & -13.301 \\
Nearest Neighbor & 0.888 & 2.039 & 0.230 & 0.670 & -17.132 \\
IDW (3 NBR) & 0.839 & 3.282 & 0.6065 & 0.540 & -15.918 \\
\hline
\end{tabular}


Table 7. Comparison of the manifold method with other standard methods for precipitation over the Grand River watershed.

\begin{tabular}{|c|c|c|c|c|c|c|}
\hline \multirow[b]{2}{*}{ Method } & \multicolumn{3}{|c|}{ USGS04116000 } & \multicolumn{3}{|c|}{ USGS04119000 } \\
\hline & NASH & RMSE & $\operatorname{APB}(\%)$ & $\mathrm{NASH}$ & RMSE & $\mathrm{APB}(\%)$ \\
\hline Nearest Neighbor & 0.56 & 37.13 & 36.47 & 0.58 & 58.15 & 30.54 \\
\hline Natural Neighbor & 0.33 & 44.52 & 54.71 & 0.36 & 71.88 & 48.72 \\
\hline Inverse Distance & 0.38 & 44.22 & 54.18 & 0.37 & 70.29 & 48.01 \\
\hline Manifold & 0.59 & 35.56 & 33.76 & 0.63 & 54.57 & 27.87 \\
\hline
\end{tabular}

Table 8. Cross-validation results for precipitation over the Grand River watershed.

\begin{tabular}{lccccc}
\hline Method & $\mathrm{R}^{2}$ & RMSE $(\mathrm{cms})$ & Fn & NASH & APB (\%) \\
\hline Manifold & 0.543 & 7.933 & 0.910 & 0.624 & 72.4 \\
Natural Neighbor & 0.543 & 7.920 & 0.901 & 0.574 & 73.6 \\
Nearest Neighbor & 0.471 & 8.802 & 0.930 & 0.604 & 81.8 \\
IDW (3 NBR) & 0.567 & 7.542 & 0.907 & 0.619 & 70.0 \\
\hline
\end{tabular}


(a)

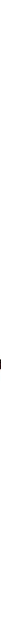

(b)

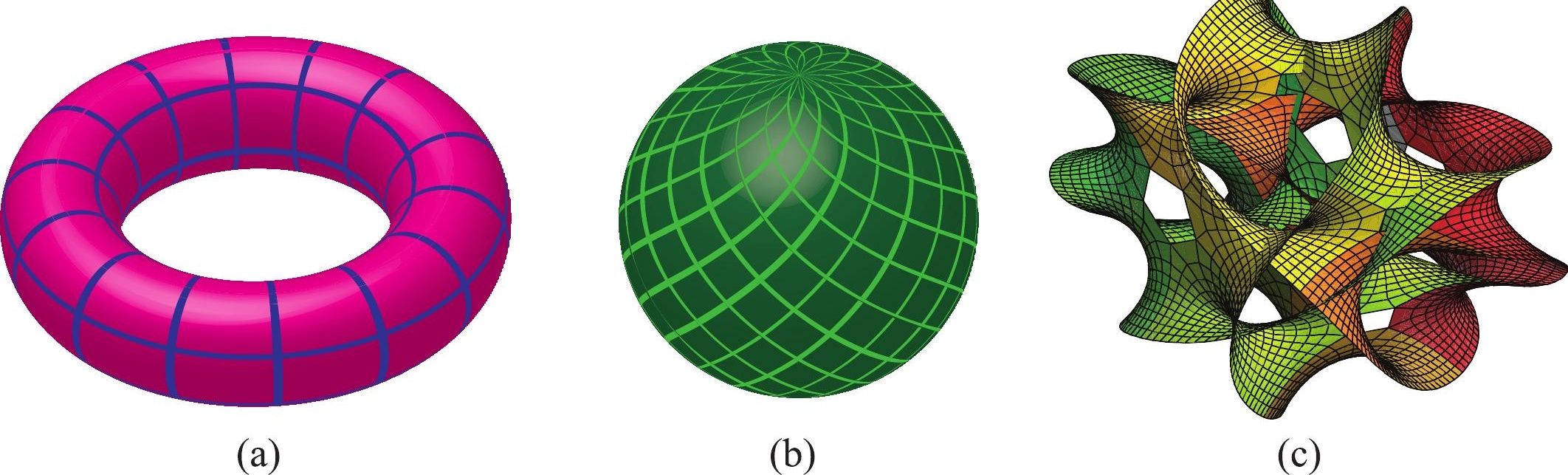

(c)

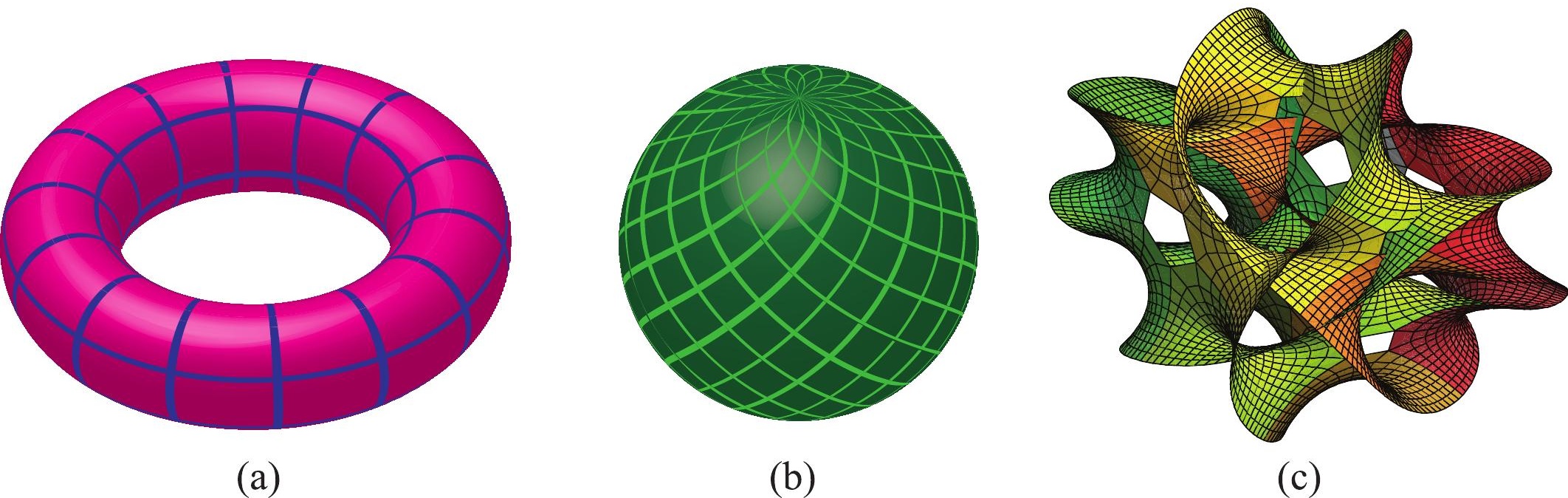

(U)




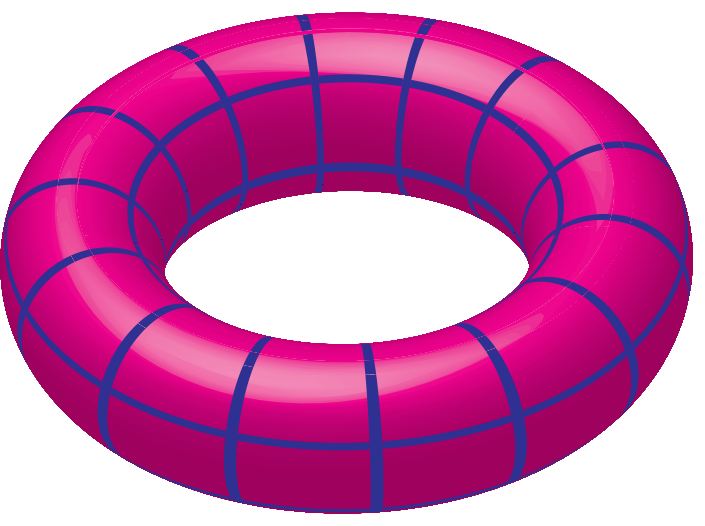

(a)

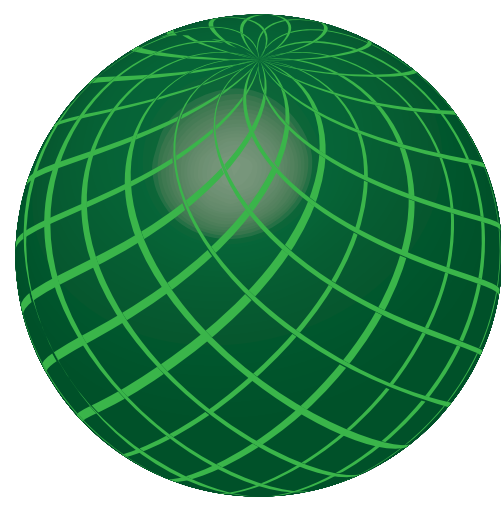

(b)

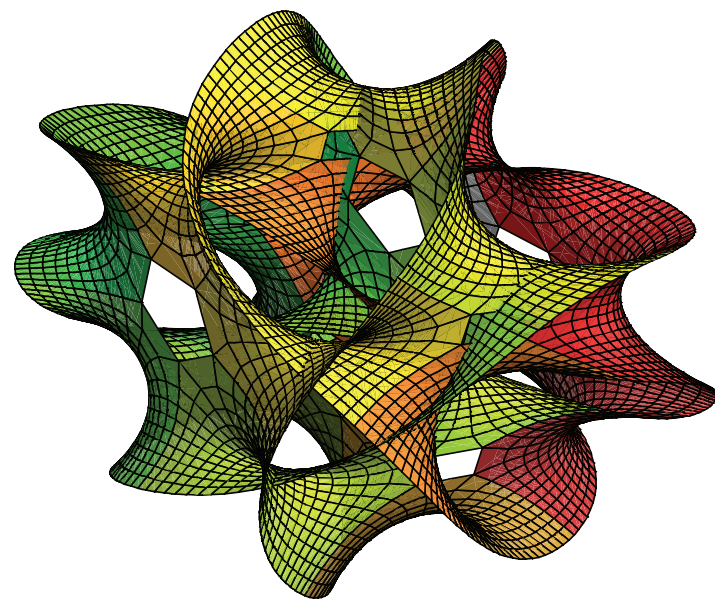

(c) 


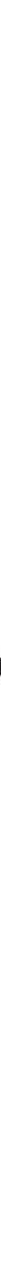

Figure 2
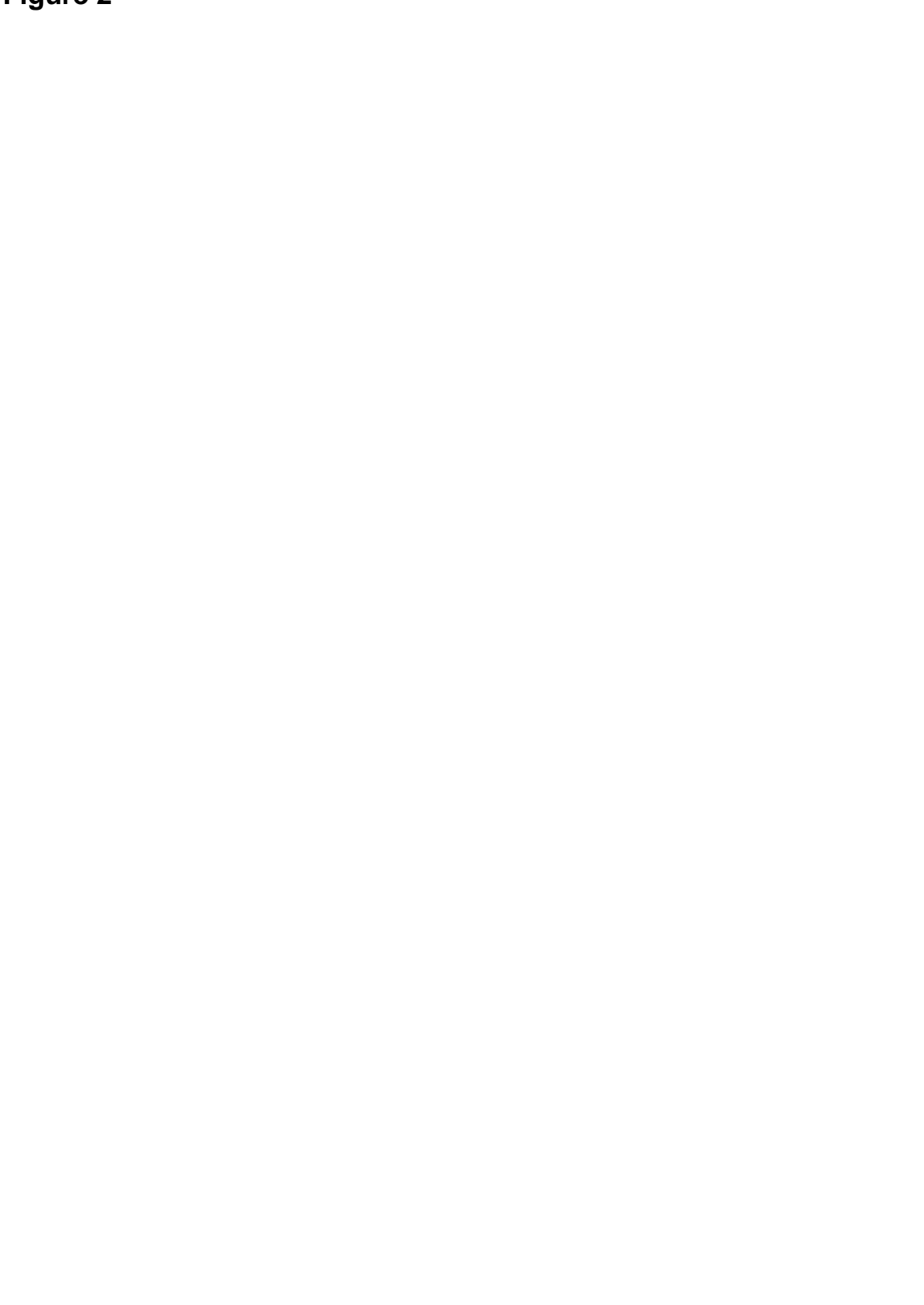
Figure 3

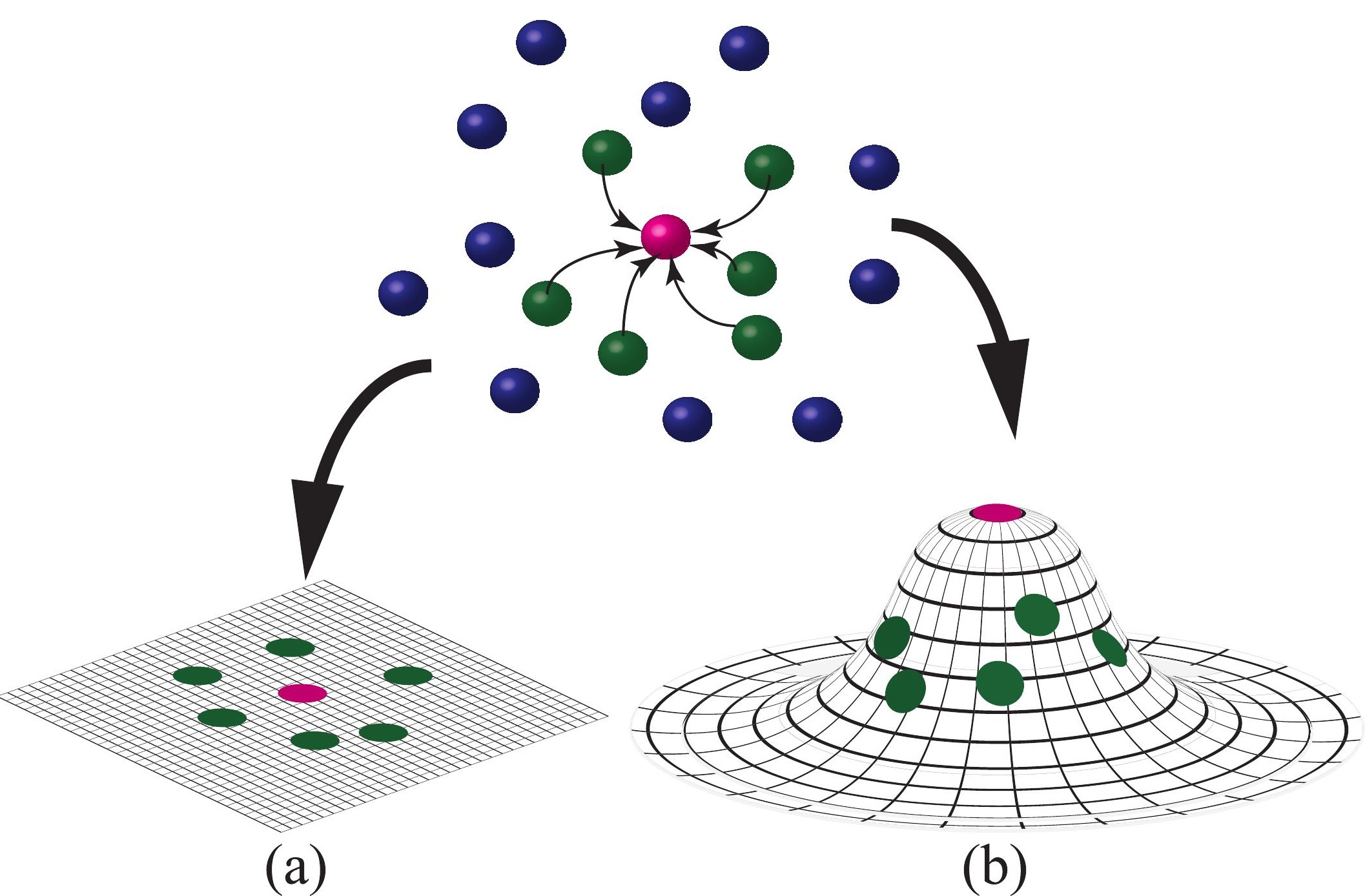

\section{Fure 3}
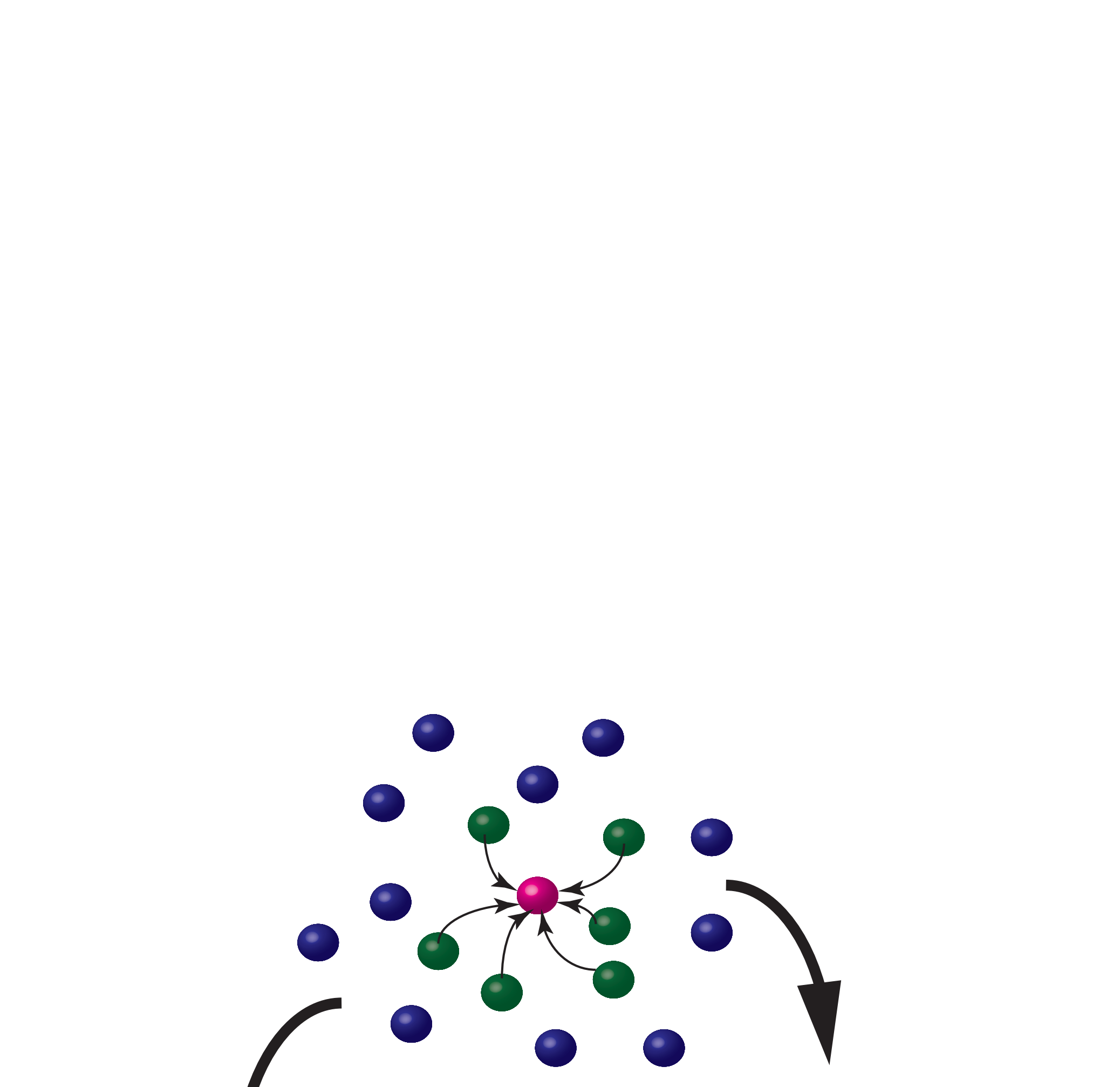
(b)

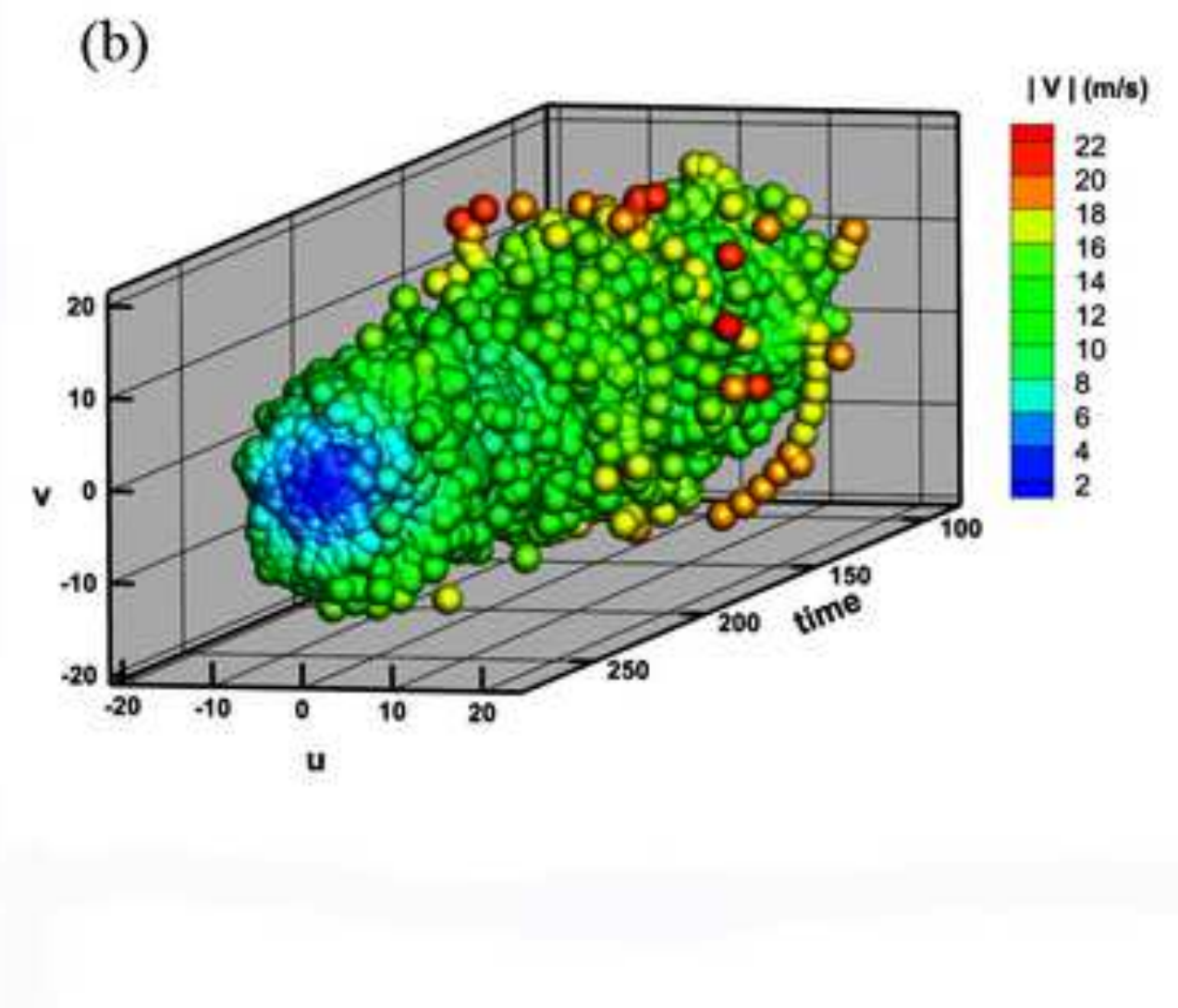

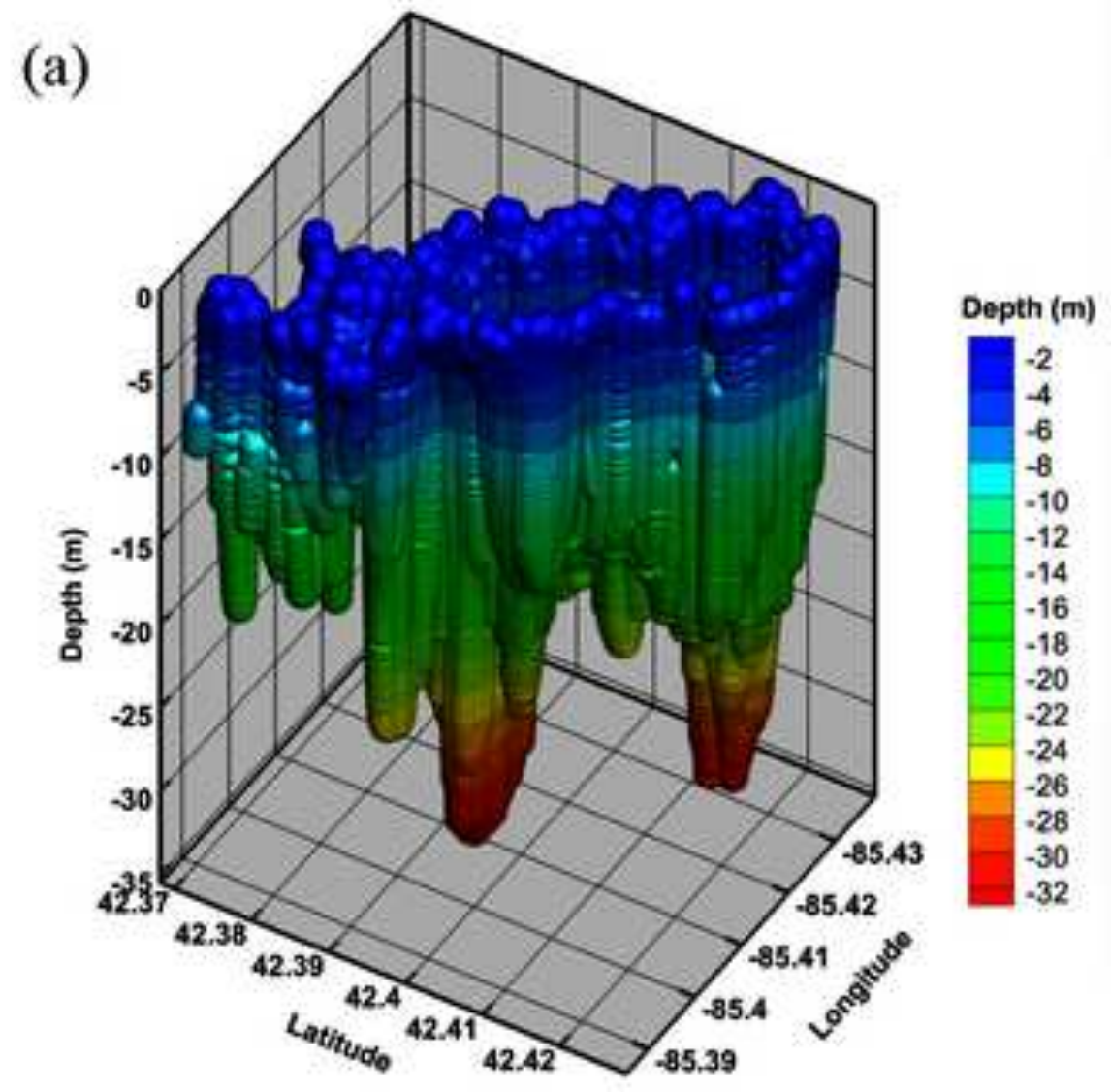

(a) 
Figure 5

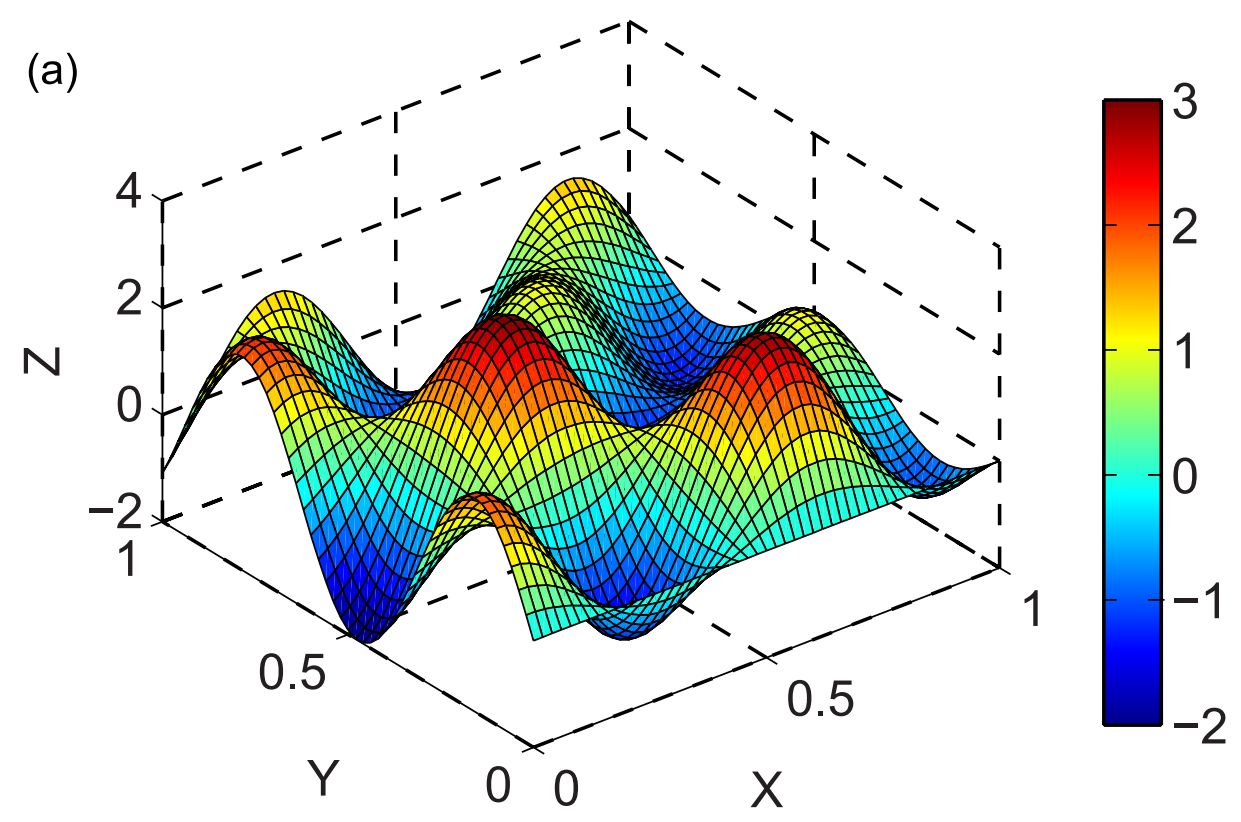

(b) 30 points

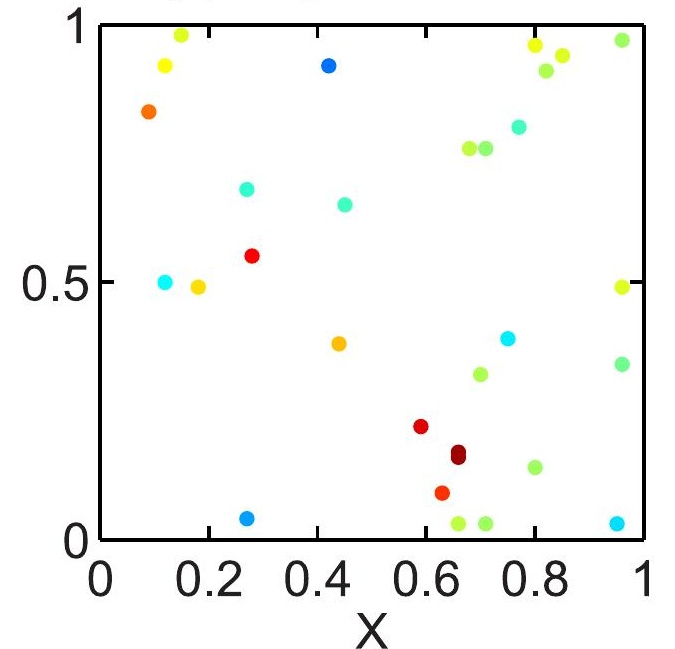

(c) 60 points

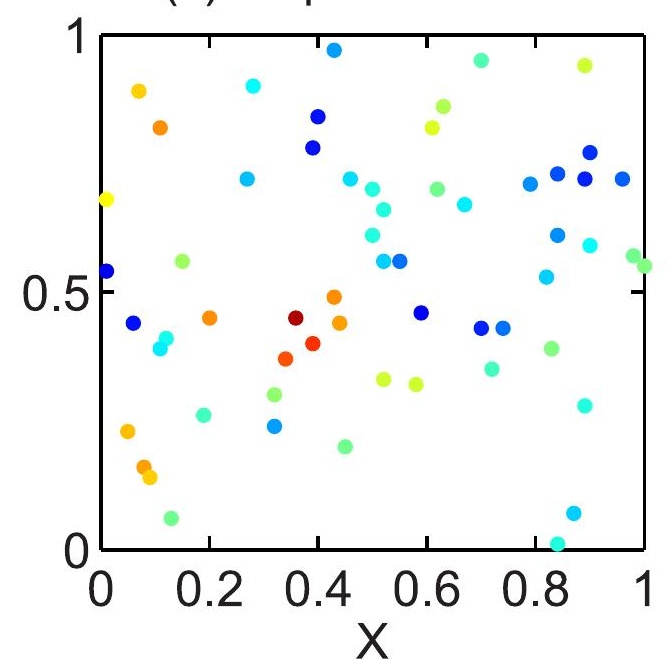

(d) 90 points

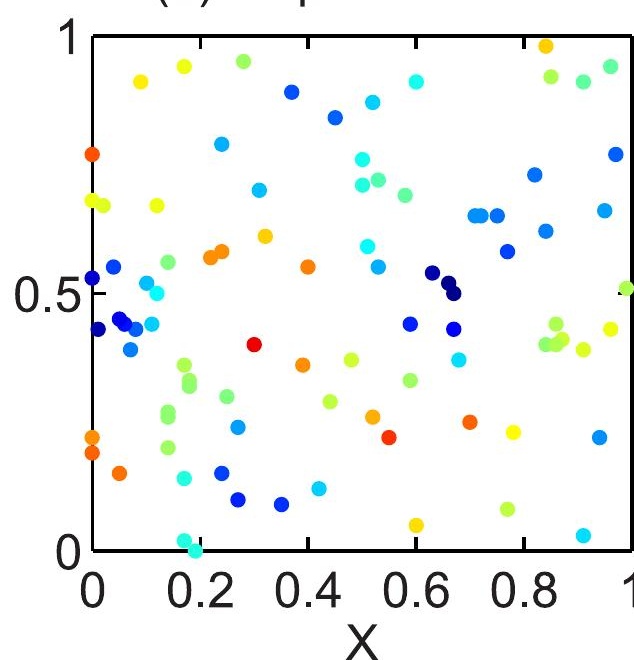


$88^{\circ} 0^{\prime} 0 " \mathrm{~W} 87^{\circ} 0^{\prime} \mathrm{O} \mathrm{W}$ 860'0"W 850'0"W

$46^{\circ} 0^{\prime} 0^{\prime \prime} \mathrm{N}-$

$45^{\circ} 0^{\prime} 0^{\prime \prime} \mathrm{N}-$

$44^{\circ} 0^{\prime} 0^{\prime \prime} \mathrm{N}-$

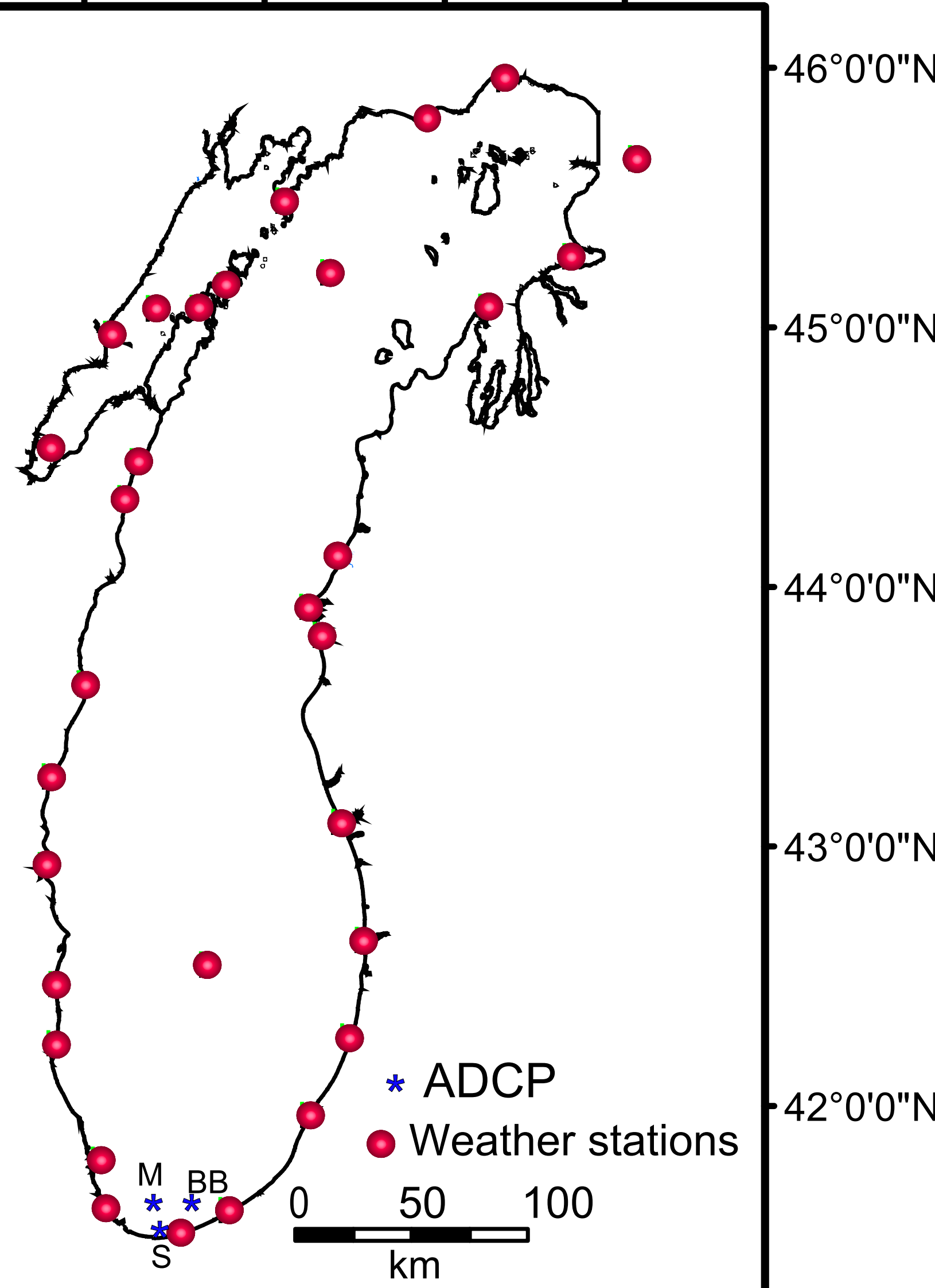

$-46^{\circ} 0^{\prime} 0^{\prime \prime} \mathrm{N}$

$45^{\circ} 0^{\prime} 0^{\prime \prime} \mathrm{N}$

$43^{\circ} 0^{\prime} 0^{\prime \prime} \mathrm{N}-$

$42^{\circ} 0^{\prime} \mathrm{O}^{\prime \prime} \mathrm{N}-$ 
(a)

Depth (m)

0.00

$-33.26$

(b)

$85^{\circ} 26^{\prime} 0 " \mathrm{~W} \quad 85^{\circ} 25^{\prime} 0 " \mathrm{~W} \quad 85^{\circ} 24^{\prime} 0^{\prime \prime} \mathrm{W} \quad 85^{\circ} 23^{\prime} 0 " \mathrm{~W}$

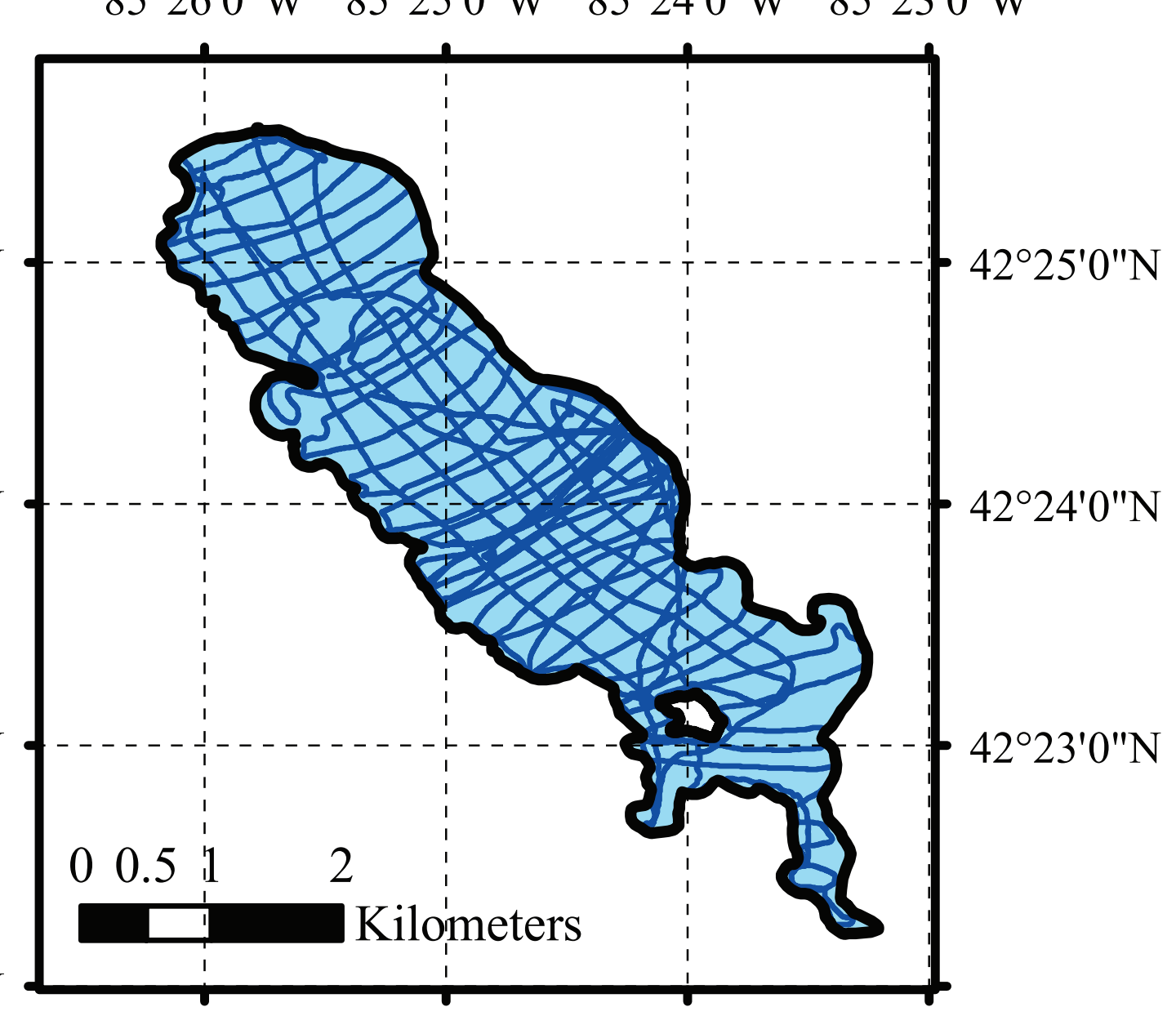

$85^{\circ} 26^{\prime} 0^{\prime \prime} \mathrm{W} \quad 85^{\circ} 25^{\prime} 0^{\prime \prime} \mathrm{W} \quad 85^{\circ} 24^{\prime} 0^{\prime \prime} \mathrm{W} \quad 85^{\circ} 23^{\prime} 0^{\prime \prime} \mathrm{W}$

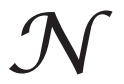

$42^{\circ} 23^{\prime} 0^{\prime \prime} \mathrm{N}$

$42^{\circ} 22^{\prime} 0^{\prime \prime} \mathrm{N}$

$42^{\circ} 25^{\prime} 0^{\prime \prime} \mathrm{N}$

$42^{\circ} 24^{\prime} 0^{\prime \prime} \mathrm{N}$

4 


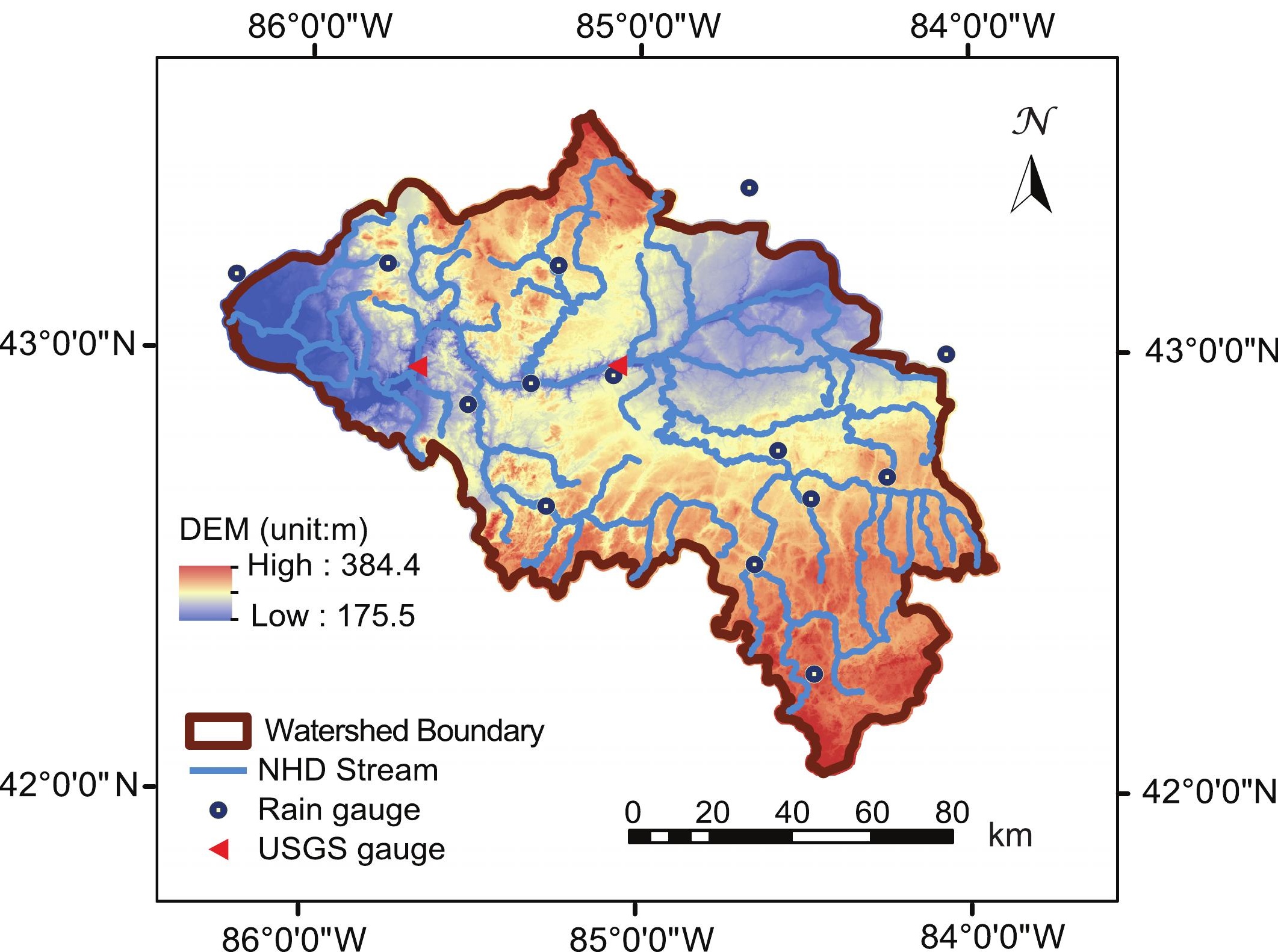


Figure 10
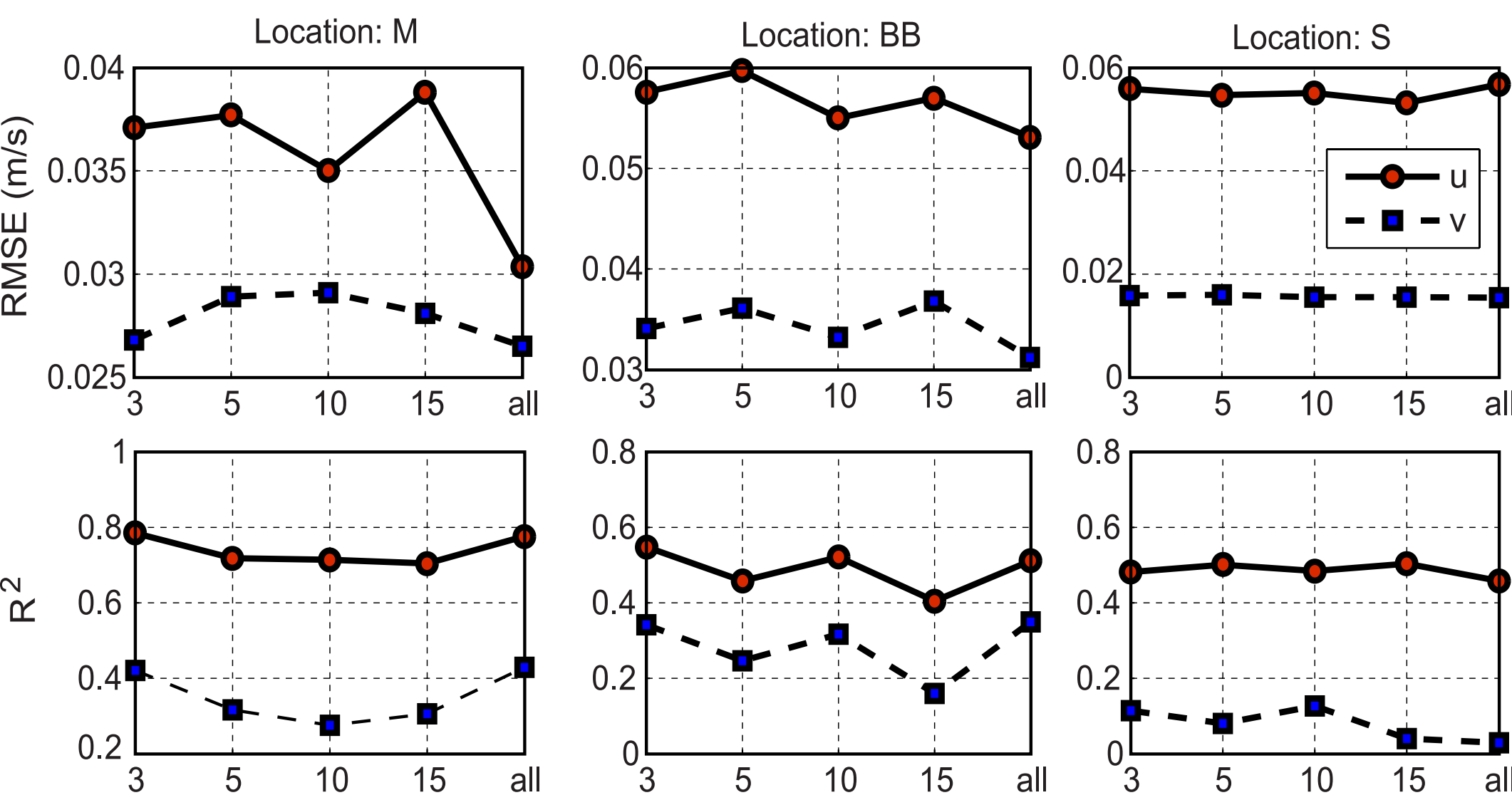

Number of nearest neighbors (k) 


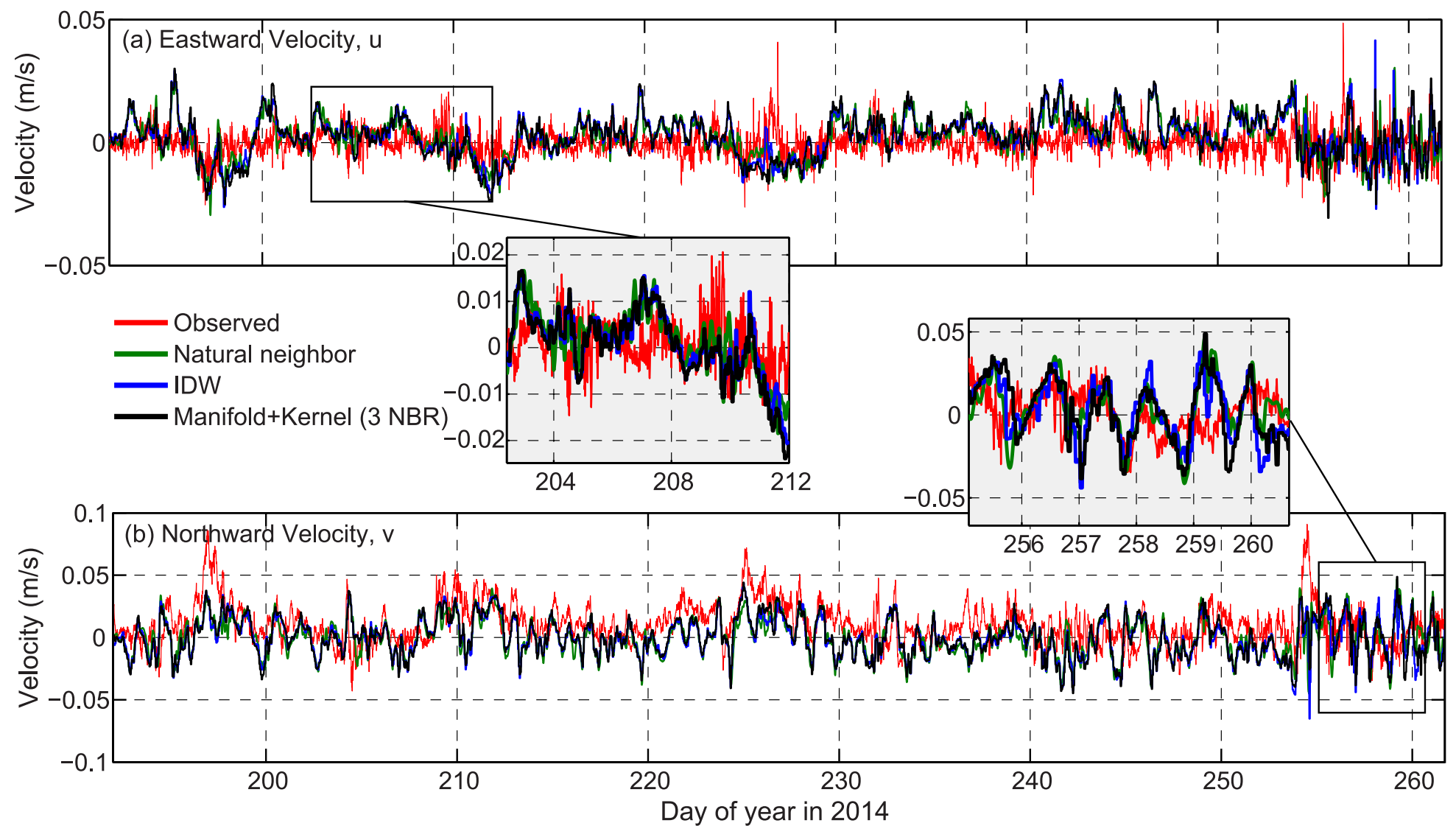


